\title{
GROUND STATES FOR FRACTIONAL KIRCHHOFF EQUATIONS WITH CRITICAL NONLINEARITY IN LOW DIMENSION
}

\author{
ZHISU LIU, MARCO SQUASSINA, AND JIANJUN ZHANG
}

\begin{abstract}
We study the existence of ground states to a nonlinear fractional Kirchhoff equation with an external potential $V$. Under suitable assumptions on $V$, using the monotonicity trick and the profile decomposition, we prove the existence of ground states. In particular, the nonlinearity does not satisfy the Ambrosetti-Rabinowitz type condition or monotonicity assumptions.
\end{abstract}

\section{CONTENTS}

1. Introduction and results 1

1.1. Overview 1

1.2. Main results 3

1.3. Main difficulties 4

2. Variational setting 5

3. The perturbed functional 6

4. Upper estimate of $c_{\lambda}$ and limit problems 9

4.1. An energy estimate 9

4.2. The limit problem 12

5. Behaviour of Palais-Smale sequences 12

5.1. Splitting lemmas 13

5.2. Profile decomposition 16

6. Proof of the main results 20

6.1. Nontrivial critical points of $I_{\lambda} \quad 20$

6.2. Completion of the proof 22

References 24

\section{IntRoduction AND RESUlts}

1.1. Overview. In this paper we are concerned with the existence of positive ground state solutions to the following nonlinear fractional Kirchhoff equation

$$
\left\{\begin{array}{r}
\left(a+b \int_{\mathbb{R}^{N}}\left|(-\Delta)^{\frac{\alpha}{2}} u\right|^{2} d x\right)(-\Delta)^{\alpha} u+V(x) u=f(u) \quad \text { in } \mathbb{R}^{N}, \\
u \in H^{\alpha}\left(\mathbb{R}^{N}\right), \quad u>0 \quad \text { in } \mathbb{R}^{N},
\end{array}\right.
$$

where $a, b$ are positive constants, $\alpha \in(0,1)$ and $N>2 \alpha$. The operator $(-\Delta)^{\alpha}$ is the fractional Laplacian defined as $\mathscr{F}^{-1}\left(|\xi|^{2 \alpha} \mathscr{F}(u)\right)$, where $\mathscr{F}$ denotes the Fourier transform on $\mathbb{R}^{N}$. When $a=1$ and $b=0$, then $(\mathrm{K})$ reduces to the following fractional Schrödinger equation

$$
(-\Delta)^{\alpha} u+V(x) u=f(u) \quad \text { in } \mathbb{R}^{N},
$$

2000 Mathematics Subject Classification. 35Q55, 35Q51, 53C35.

Key words and phrases. Fractional Kirchhoff type problems, ground state solutions, profile decomposition.

Z. Liu is supported by the NSFC(11626127). M. Squassina is member of the Gruppo Nazionale per l'Analisi Matematica, la Probabilità e le loro Applicazioni (GNAMPA) of the Istituto Nazionale di Alta Matematica (INdAM). J. J. Zhang was partially supported by the Science Foundation of Chongqing Jiaotong University(15JDKJC-B033). 
which has been proposed by Laskin [18] in fractional quantum mechanics as a result of extending the Feynman integrals from the Brownian like to the Lévy like quantum mechanical paths. For such a class of fractional and nonlocal problems, Caffarelli and Silvestre [8] expressed $(-\Delta)^{\alpha}$ as a Dirichlet-Neumann map for a certain local elliptic boundary value problem on the half-space. This method is a valid tool to deal with equations involving fractional operators to get regularity and handle variational methods. We refer the readers to $[14,31]$ and to the references therein. Investigated first in $[11,12]$ via variational methods, there has been a lot of interest in the study of the existence and multiplicity of solutions for (1.1) when $V$ and $f$ satisfy general conditions. We cite $[10,29,30]$ with no attempts to provide a complete list of references.

If $\alpha=1$, then problem (K) formally reduces to the well-known Kirchhoff equation

$$
-\left(a+b \int_{\mathbb{R}^{N}}|\nabla u|^{2} d x\right) \Delta u+V(x) u=f(u) \text { in } \mathbb{R}^{N},
$$

related to the stationary analogue of the Kirchhoff-Schrödinger type equation

$$
\frac{\partial^{2} u}{\partial t^{2}}-\left(a+b \int_{\Omega}|\nabla u|^{2} d x\right) \Delta u=f(t, x, u)
$$

where $\Omega$ is a bounded domain in $\mathbb{R}^{N}, u$ denotes the displacement, $f$ is the external force, $b$ is the initial tension and $a$ is related to the intrinsic properties of the string. Equations of this type were first proposed by Kirchhoff [17] in 1883 to describe the transversal oscillations of a stretched string. Besides, we also point out that such nonlocal problems appear in other fields like biological systems, where $u$ describes a process depending on the average of itself. We refer readers to Chipot and Lovat [9], Alves et al. [1]. However, the solvability of the Kirchhoff type equations has been well studied in a general dimension by various authors only after J.-L. Lions [21] introduced an abstract framework to such problems. For more recent results concerning Kirchhoff-type equations we refer e.g. to $[4,15,24,26,35,37]$.

In [20], by using a monotonicity trick and a global compactness lemma, Li and Ye proved that for $f(u)=|u|^{p-2} u$ and $p \in(3,2 N /(N-2))$, problem (1.2) has a positive ground state. Subsequently, Liu and Guo [23] extended the above result to $p \in(2,2 N /(N-2))$. Fiscella and Valdinoci, in [13], proposed the following stationary Kirchhoff variational equation with critical growth

$$
\left\{\begin{array}{l}
M\left(\int_{\mathbb{R}^{N}}\left|(-\Delta)^{\frac{\alpha}{2}} u\right|^{2} d x\right)(-\Delta)^{\alpha} u=\lambda f(x, u)+|u|^{2_{\alpha}^{*}-2} u \text { in } \Omega, \\
u=0 \text { in } \mathbb{R}^{N} \backslash \Omega
\end{array}\right.
$$

which models nonlocal aspects of the tension arising from measurements of the fractional length of the string. They obtained the existence of non-negative solutions when $M$ and $f$ are continuous functions satisfying suitable assumptions. Autuori, Fiscella and Pucci [3] considered the existence and the asymptotic behavior of non-negative solutions of (1.3). Pucci and Saldi [27] established multiplicity of nontrivial solutions. Via a three critical points theorem, Nyamoradi [25] studied the subcritical case of (1.3) and obtained three solutions. See also $[28,36]$ for related results.

To the best of our knowledge, there are few papers in the literature on fractional Kirchhoff equations in $\mathbb{R}^{N}$. Recently, Ambrosio and Isernia [2] considered the fractional Kirchhoff problem

$$
\left(a+b \int_{\mathbb{R}^{N}}\left|(-\Delta)^{\frac{\alpha}{2}} u\right|^{2} d x\right)(-\Delta)^{\alpha} u=f(u) \quad \text { in } \mathbb{R}^{N}
$$

where $f$ is an odd subcritical nonlinearity satisfying the well known Berestycki-Lions assumptions [6]. By minimax arguments, the authors establish a multiplicity result in the radial space $H_{\text {rad }}^{\alpha}\left(\mathbb{R}^{N}\right)$ 
when the parameter $b$ is sufficiently small. As in [20], Teng [33] also searched for ground state solutions for the fractional Schrödinger-Poisson system in $\mathbb{R}^{3}$ with critical growth

$$
\begin{cases}(-\Delta)^{\alpha} u+V(x) u+\phi u=\mu|u|^{q-2} u+|u|^{2_{\alpha}^{*}-2} u & \text { in } \mathbb{R}^{3} \\ (-\Delta)^{t} \phi=u^{2} & \text { in } \mathbb{R}^{3} .\end{cases}
$$

We point out that, in $[20,33]$ the corresponding limit problems play an important rǒle. In order to get the existence of ground state solutions of the limit problems, the authors used a constrained minimization on a manifold $\mathcal{M}$ obtained by combining the Nehari and Pohožaev manifolds.

1.2. Main results. Motivated by the works above, in this paper we aim to study the existence of positive ground state solutions to the fractional Kirchhoff equation with the Berestycki-Lions type conditions of critical type, firstly introduced in [38].

1.2.1. Assumptions on $V$. On the external potential we assume the following:

$\left(\mathrm{V}_{1}\right) V \in C^{1}\left(\mathbb{R}^{N}, \mathbb{R}\right)$ and, setting $W(x):=\max \{x \cdot \nabla V(x), 0\}$, we assume

$$
\|W\|_{L^{\frac{N}{2 \alpha}\left(\mathbb{R}^{N}\right)}}<2 a \alpha S_{\alpha}, \quad S_{\alpha}:=\inf _{\substack{u \in D^{\alpha, 2}\left(\mathbb{R}^{N}\right) \\ u \neq 0}} \frac{\int_{\mathbb{R}^{N}}\left|(-\Delta)^{\frac{\alpha}{2}} u\right|^{2} d x}{\left(\int_{\mathbb{R}^{N}}|u|^{2_{\alpha}^{*}} d x\right)^{2 / 2_{\alpha}^{*}}}, \quad 2_{\alpha}^{*}:=\frac{2 N}{N-2 \alpha} ;
$$

$\left(\mathrm{V}_{2}\right)$ there exists $V_{\infty} \in \mathbb{R}$ such that

$$
V(x) \leq \lim _{|y| \rightarrow \infty} V(y)=V_{\infty}, \quad \text { for all } x \in \mathbb{R}^{N}
$$

$\left(\mathrm{V}_{3}\right)$ the operator $a(-\Delta)^{\alpha}+V(x): H^{\alpha}\left(\mathbb{R}^{N}\right) \rightarrow H^{-\alpha}\left(\mathbb{R}^{N}\right)$ satisfies

$$
\inf _{\substack{u \in H^{\alpha}\left(\mathbb{R}^{N}\right) \\ u \neq 0}} \frac{\int_{\mathbb{R}^{N}}\left(a\left|(-\Delta)^{\frac{\alpha}{2}} u\right|^{2}+V(x) u^{2}\right) d x}{\int_{\mathbb{R}^{N}}|u|^{2} d x}>0 .
$$

1.2.2. Assumptions on $f$. We assume that $f(t)=0$ for all $t \leq 0$ and

$\left(\mathrm{f}_{1}\right) f \in C^{1}\left(\mathbb{R}^{+}, \mathbb{R}\right)$ and $\lim _{t \rightarrow 0} \frac{f(t)}{t}=0$;

$\left(\mathrm{f}_{2}\right) \lim _{t \rightarrow \infty} \frac{f(t)}{t^{2 *}-1}=1$;

$\left(\mathrm{f}_{3}\right)$ there are $D>0$ and $2<q<2_{\alpha}^{*}$ such that $f(t) \geq t^{2_{\alpha}^{*}-1}+D t^{q-1}$ for any $t \geq 0$.

Now we state our first result.

Theorem 1.1. Assume $\left(\mathrm{V}_{1}\right)-\left(\mathrm{V}_{3}\right),\left(\mathrm{f}_{1}\right)-\left(\mathrm{f}_{3}\right)$ and $N=2$ with $\alpha \in\left(\frac{1}{2}, 1\right)$ or $N=3$ with $\alpha \in\left(\frac{3}{4}, 1\right)$.

(i) If $q \in\left(2,2_{\alpha}^{*}\right)$, there is $D_{1}>0$ such that, for $D \geq D_{1}$, (K) admits a positive ground state solution.

(ii) If $q \in\left(\frac{4 \alpha}{N-2 \alpha}, 2_{\alpha}^{*}\right)$, for any $D>0,(\mathrm{~K})$ admits a positive ground state solution.

We point out that without any symmetry assumption on $V$, the ground state solution obtained above maybe is not radially symmetric. In the following, we impose a monotonicity assumption of $V$ and show that $(\mathrm{K})$ admits a radially symmetric solution.

Assume now that $V$ is radially symmetric and increasing, that is

$$
\text { for all } x, y \in \mathbb{R}^{N}:|x| \leq|y| \Rightarrow V(x) \leq V(y) .
$$

Theorem 1.2. Under the assumptions of Theorem 1.1 and $\left(V_{4}\right),(\mathrm{K})$ admits a radially symmetric positive solution at the global (unrestricted to radial paths) mountain pass energy level. 
As a main tool to prove Theorem 1.1 we shall give the profile decomposition of the Palais-Smale sequences by which we can derive some compactness and get a positive ground states for (K). The main tool for the proof of Theorem 1.2 is a symmetric version of the monotonicity trick [32]. We recall that Zhang and Zou [39] studied the critical case for Berestycki-Lions theorem of the Schrödinger equation $-\Delta u+V(x) u=f(u)$. They obtained positive ground state solutions when $V$ satisfies similar assumptions as $\left(\mathrm{V}_{1}\right)-\left(\mathrm{V}_{3}\right), f$ satisfies $\left(\mathrm{f}_{1}\right)-\left(\mathrm{f}_{3}\right)$ and

$\left(\mathrm{f}_{4}\right)\left|f^{\prime}(t)\right| \leq C\left(1+|t|^{\frac{4}{N-2}}\right)$, for $t>0$ and some $C>0$.

We should mention that in the present paper, $\left(\mathrm{f}_{4}\right)$ is removed.

1.3. Main difficulties. We mention the difficulties and the idea in proving Theorem 1.1.

Firstly, without the Ambrosetti-Rabinowitz condition, it is difficult to get the boundedness of Palais-Smale sequences. In order to overcome this difficulty, inspired by [20], we will use the monotonicity trick developed by Jeanjean [16], introduce a family of functionals $I_{\lambda}$ and obtain a bounded $(\mathrm{PS})_{c_{\lambda}}$ sequence for $I_{\lambda}$ for almost all $\lambda$ in an interval $J$, where $c_{\lambda}$ is given in Section 3 .

Secondly, by the presence of the Kirchhoff term, one obstacle arises in getting the compactness of $I_{\lambda}$, even in the subcritical case. Precisely, this does not hold in general: for any $\varphi \in C_{0}^{\infty}\left(\mathbb{R}^{N}\right)$,

$$
\int_{\mathbb{R}^{N}}\left|(-\Delta)^{\frac{\alpha}{2}} u_{n}\right|^{2} d x \int_{\mathbb{R}^{N}}(-\Delta)^{\frac{\alpha}{2}} u_{n}(-\Delta)^{\frac{\alpha}{2}} \varphi d x \rightarrow \int_{\mathbb{R}^{N}}\left|(-\Delta)^{\frac{\alpha}{2}} u\right|^{2} d x \int_{\mathbb{R}^{N}}(-\Delta)^{\frac{\alpha}{2}} u(-\Delta)^{\frac{\alpha}{2}} \varphi d x,
$$

where $\left\{u_{n}\right\}_{n \in \mathbb{N}}$ is a (PS)-sequence of $I_{\lambda}$ satisfying $u_{n} \rightarrow u$ in $H^{\alpha}\left(\mathbb{R}^{N}\right)$. Then, even in the subcritical case, it is not clear that weak limits are critical points of $I_{\lambda}$. In [2], for (1.4) the compactness was recovered by restricting $I_{\lambda}$ to the radial space $H_{\text {rad }}^{\alpha}\left(\mathbb{R}^{N}\right)$, which is compactly embedded $L^{s}\left(\mathbb{R}^{N}\right)$ for all $s \in\left(2,2_{\alpha}^{*}\right)$. For the related works in the bounded domains, see e.g. [13,27,36].

In the present paper, we do not impose any symmetry and just consider $(\mathrm{K})$ in $H^{\alpha}\left(\mathbb{R}^{N}\right)$. So the arguments mentioned above cannot be applied. Inspired by [20], in place of $I_{\lambda}$, we consider a family of related functionals $J_{\lambda}$, whose corresponding problem is a non Kirchhoff equation.

Thirdly, the critical exponent makes the problem rather tough. The (PS)-condition does not hold in general and to overcome this difficulty, we show that the mountain pass level $c_{\lambda}$ is strictly less than some critical level $c_{\lambda}^{*}$. For $-\Delta u+V(x) u=\lambda f(u)$ with critical growth, if $S$ is the best constant of $D^{1,2}\left(\mathbb{R}^{N}\right) \hookrightarrow L^{2^{*}}\left(\mathbb{R}^{N}\right)$, one can show that [7]

$$
c_{\lambda}^{*}=\frac{1}{N} S^{\frac{N}{2}} \lambda^{\frac{2-N}{2}} .
$$

For $-\left(a+b \int_{\mathbb{R}^{3}}|\nabla u|^{2} d x\right) \Delta u+V(x) u=\lambda f(u)$ in $\mathbb{R}^{3}$ involving critical growth [19,22]

$$
c_{\lambda}^{*}=\frac{a b}{4 \lambda} S^{3}+\frac{\left[b^{2} S^{4}+4 \lambda a S\right]^{\frac{3}{2}}}{24 \lambda^{2}}+\frac{b^{3} S^{6}}{24 \lambda^{2}} .
$$

However, for fractional Kirchhoff equations, to give the exact value of $c_{\lambda}^{*}$ is complicated, since one cannot solve precisely a fractional order algebra equation. A careful analysis is needed at this stage. With an estimate of $c_{\lambda}$, inspired by [39], we establish a profile decomposition of the Palais-Smale sequence $\left\{u_{n}\right\}_{n \in \mathbb{N}}$ (Lemma 5.4) related to $J_{\lambda}$. Thanks to this result, for almost every $\lambda \in[1 / 2,1]$ we obtain a nontrivial critical point $u_{\lambda}$ of $I_{\lambda}$ at the level $c_{\lambda}$. Finally, choosing a sequence $\lambda_{n} \subset[1 / 2,1]$ with $\lambda_{n} \rightarrow 1$, thanks to the Pohožaev identity we obtain a bounded $(P S)_{c_{1}}$-sequence of the original functional $I$. Using the idea above again, we obtain a nontrivial solution of problem $(\mathrm{K})$.

Throughout this paper, $C$ will denote a generic positive constant.

The paper is organized as follows.

In Section 2, the variational setting and some preliminary lemmas are presented. 
In Section 3, we consider a perturbation of the original problem $(\mathrm{K})$. Then using the monotonicity trick developed by Jeanjean, we obtain the bounded (PS) $)_{c_{\lambda}}$-sequence $\left\{u_{n}\right\}_{n \in \mathbb{N}}$ for almost all $\lambda$. In Section 4, an upper estimate of the mountain pass value is obtained and the limit problem is discussed. In Section 5, we give the profile decomposition of $\left\{u_{n}\right\}_{n \in \mathbb{N}}$. In Section 6, Theorem 1.1 and 1.2 are finally proved.

\section{VARIATIONAL SETTING}

In this section we outline the variational framework for $(\mathrm{K})$ and recall some preliminary lemmas. For any $\alpha \in(0,1)$, the fractional Sobolev space $H^{\alpha}\left(\mathbb{R}^{3}\right)$ is defined by

$$
H^{\alpha}\left(\mathbb{R}^{N}\right):=\left\{u \in L^{2}\left(\mathbb{R}^{N}\right): \frac{|u(x)-u(y)|}{|x-y|^{\frac{N+2 \alpha}{2}}} \in L^{2}\left(\mathbb{R}^{N} \times \mathbb{R}^{N}\right)\right\} .
$$

It is known that

$$
\int_{\mathbb{R}^{2 N}} \frac{|u(x)-u(y)|^{2}}{|x-y|^{N+2 \alpha}} d x d y=2 C(n, \alpha)^{-1} \int_{\mathbb{R}^{N}}\left|(-\Delta)^{\frac{\alpha}{2}} u\right|^{2} d x
$$

where

$$
C(n, \alpha)=\left(\int_{\mathbb{R}^{N}} \frac{1-\cos \zeta_{1}}{|\zeta|^{N+2 \alpha}} d \zeta\right)^{-1}
$$

We endow the space $H^{\alpha}\left(\mathbb{R}^{N}\right)$ with the norm

$$
\|u\|_{H^{\alpha}\left(\mathbb{R}^{N}\right)}:=\left(\int_{\mathbb{R}^{N}}|u|^{2} d x+\int_{\mathbb{R}^{N}}\left|(-\Delta)^{\frac{\alpha}{2}} u\right|^{2} d x\right)^{1 / 2} .
$$

$H^{\alpha}\left(\mathbb{R}^{N}\right)$ is also the completion of $C_{0}^{\infty}\left(\mathbb{R}^{N}\right)$ with $\|\cdot\|_{H^{\alpha}\left(\mathbb{R}^{N}\right)}$ and it is continuously embedded into $L^{q}\left(\mathbb{R}^{N}\right)$ for $q \in\left[2,2_{\alpha}^{*}\right]$. The homogeneous space $D^{\alpha, 2}\left(\mathbb{R}^{N}\right)$ is

$$
D^{\alpha, 2}\left(\mathbb{R}^{N}\right):=\left\{u \in L^{2_{\alpha}^{*}}\left(\mathbb{R}^{N}\right): \frac{|u(x)-u(y)|}{|x-y|^{\frac{N+2 \alpha}{2}}} \in L^{2}\left(\mathbb{R}^{N} \times \mathbb{R}^{N}\right)\right\},
$$

and it is also the completion of $C_{0}^{\infty}\left(\mathbb{R}^{N}\right)$ with respect to the norm

$$
\|u\|_{D^{\alpha, 2}\left(\mathbb{R}^{N}\right)}:=\left(\int_{\mathbb{R}^{N}}\left|(-\Delta)^{\frac{\alpha}{2}} u\right|^{2} d x\right)^{1 / 2} .
$$

Lemma 2.1. Assume that $\left(\mathrm{V}_{2}\right)-\left(\mathrm{V}_{3}\right)$ hold. Then, for every $\varepsilon>0$ there exists $\omega_{\varepsilon}>0$ such that

$$
\int_{\mathbb{R}^{N}}\left((a-\varepsilon)\left|(-\Delta)^{\frac{\alpha}{2}} u\right|^{2}+V(x) u^{2}\right) d x \geq \omega_{\varepsilon} \int_{\mathbb{R}^{N}}|u|^{2} d x
$$

for every $u \in H^{\alpha}\left(\mathbb{R}^{N}\right)$.

Proof. By contradiction, let $\left\{\varepsilon_{n}\right\}_{n \in \mathbb{N}} \subset \mathbb{R}^{+}$with $\varepsilon_{n} \rightarrow 0$ and $\left\{u_{n}\right\}_{n \in \mathbb{N}} \subset H^{\alpha}\left(\mathbb{R}^{N}\right)$ with

$$
\int_{\mathbb{R}^{N}}\left(\left(a-\varepsilon_{n}\right)\left|(-\Delta)^{\frac{\alpha}{2}} u_{n}\right|^{2}+V(x) u_{n}^{2}\right) d x \leq \frac{1}{n} \int_{\mathbb{R}^{N}}\left|u_{n}\right|^{2} d x .
$$

Then, up to a standard nomalization, we may assume that $\left\|u_{n}\right\|_{H^{\alpha}\left(\mathbb{R}^{N}\right)}=1$ and

$$
\int_{\mathbb{R}^{N}}\left(\left(a-\varepsilon_{n}\right)\left|(-\Delta)^{\frac{\alpha}{2}} u_{n}\right|^{2}+V(x) u_{n}^{2}\right) d x \leq \frac{1}{n} .
$$

In view of $\left(\mathrm{V}_{3}\right)$, we get $\left\|u_{n}\right\|_{2} \rightarrow 0$, which implies from $\left(\mathrm{V}_{2}\right)$ and the above inequality that $\left\{u_{n}\right\}_{n \in \mathbb{N}}$ goes to zero in $D^{\alpha, 2}\left(\mathbb{R}^{N}\right)$. Therefore $u_{n} \rightarrow 0$ in $H^{\alpha}\left(\mathbb{R}^{N}\right)$, which is a contradiction. 
Let

$$
\mathcal{H}:=\left\{u \in H^{\alpha}\left(\mathbb{R}^{N}\right): \int_{\mathbb{R}^{N}} V(x) u^{2} d x<\infty\right\}
$$

be the Hilbert space equipped with the inner product

$$
\langle u, v\rangle_{\mathcal{H}}:=a \int_{\mathbb{R}^{N}}(-\Delta)^{\frac{\alpha}{2}} u(-\Delta)^{\frac{\alpha}{2}} v d x+\int_{\mathbb{R}^{N}} V(x) u v d x
$$

and the corresponding induced norm

$$
\|u\|:=\left(\int_{\mathbb{R}^{N}} a\left|(-\Delta)^{\frac{\alpha}{2}} u\right|^{2} d x+\int_{\mathbb{R}^{N}} V(x) u^{2} d x\right)^{1 / 2} .
$$

From Lemma 2.1, it easily follows that the above norm is equivalent to $\|\cdot\|_{H^{\alpha}}$. A function $u \in \mathcal{H}$ is a (weak) solution to problem $(\mathrm{K})$ if, for every $\varphi \in \mathcal{H}$, we have

$$
\left(a+b \int_{\mathbb{R}^{N}}\left|(-\Delta)^{\frac{\alpha}{2}} u\right|^{2} d x\right) \int_{\mathbb{R}^{N}}(-\Delta)^{\alpha / 2} u(-\Delta)^{\alpha / 2} \varphi d x+\int_{\mathbb{R}^{N}} V(x) u \varphi d x=\int_{\mathbb{R}^{N}} f(u) \varphi d x .
$$

We stress that, under assumptions $\left(V_{1}\right)-\left(V_{3}\right)$ and $\left(f_{1}\right)-\left(f_{3}\right)$, if $u$ is a weak solution to the above problem, then $u$ is globally bounded and Hölder regular allowing the pointwise reppresentation of $(-\Delta)^{\alpha} u$ by the results of [12]. In particular $u>0$ a.e. wherever $u \geq 0$.

Lemma 2.2 (Lions lemma, see [29]). Assume that $\left\{u_{n}\right\}_{n \in \mathbb{N}}$ is bounded in $\mathcal{H}$ and

$$
\lim _{n \rightarrow \infty} \sup _{y \in \mathbb{R}^{N}} \int_{B_{r}(y)}\left|u_{n}\right|^{2} d x=0,
$$

for some $r>0$. Then $u_{n} \rightarrow 0$ in $L^{s}\left(\mathbb{R}^{N}\right)$ for all $s \in\left(2,2_{\alpha}^{*}\right)$.

The energy functional associated with $(\mathrm{K}), I: \mathcal{H} \rightarrow \mathbb{R}$, is defined as

$$
I(u)=\frac{1}{2}\|u\|^{2}+\frac{b}{4}\left(\int_{\mathbb{R}^{N}}\left|(-\Delta)^{\frac{\alpha}{2}} u\right|^{2} d x\right)^{2}-\int_{\mathbb{R}^{N}} F(u) d x, \quad u \in \mathcal{H},
$$

with $F(u)=\int_{0}^{u} f(t) d t$. Obviously $I \in C^{1}(\mathcal{H})$ and its critical points are weak solutions to $(\mathrm{K})$.

\section{The Perturbed FunCtional}

Since we do not impose the well-known Ambrosetti-Rabinowitz condition, the boundedness of the Palais-Smale sequence becomes complicated. To overcome this difficulty, we adopt a monotonicity trick due to Jeanjean [16].

Theorem 3.1 (Monotonicity trick $[16])$. Let $(E,\|\cdot\|)$ be a real Banach space with its dual space $E^{\prime}$ and $J \in \mathbb{R}^{+}$an interval. Consider the family of $C^{1}$ functionals on $E$

$$
I_{\lambda}=A(u)-\lambda B(u), \forall \lambda \in J,
$$

with $B$ nonnegative and either $A(u) \rightarrow+\infty$ or $B(u) \rightarrow+\infty$ as $\|u\| \rightarrow \infty$, satisfying $I_{\lambda}(0)=0$. We set

$$
\Gamma_{\lambda}:=\left\{\gamma \in C([0,1], E) \mid \gamma(0)=0, I_{\lambda}(\gamma(1))<0\right\}, \quad \text { for all } \lambda \in J .
$$

If for every $\lambda \in J, \Gamma_{\lambda}$ is nonempty and

$$
c_{\lambda}=\inf _{\gamma \in \Gamma_{\lambda}} \max _{s \in[0,1]} I_{\lambda}(\gamma(s))>0,
$$

then for almost any $\lambda \in J, I_{\lambda}$ admits a bounded Palais-Smale sequence $\left\{u_{n}\right\}_{n \in \mathbb{N}} \subset E$, namely $\sup _{n \in \mathbb{N}}\left\|u_{n}\right\|<\infty, I_{\lambda}\left(u_{n}\right) \rightarrow c_{\lambda}$ and $I_{\lambda}^{\prime}\left(u_{n}\right) \rightarrow 0$ in $E^{\prime}$. Moreover $\lambda \rightarrow c_{\lambda}$ is left continuous. 
Set $J:=\left[\frac{1}{2}, 1\right], E:=\mathcal{H}$ and

$$
A(u):=\frac{1}{2}\|u\|^{2}+\frac{b}{4}\left(\int_{\mathbb{R}^{N}}\left|(-\Delta)^{\frac{\alpha}{2}} u\right|^{2} d x\right)^{2}, \quad B(u):=\int_{\mathbb{R}^{N}} F(u) d x .
$$

We consider the family of functionals $I_{\lambda}: \mathcal{H} \rightarrow \mathbb{R}$ defined by $I_{\lambda}(u)=A(u)-\lambda B(u)$, that is

$$
I_{\lambda}(u)=\frac{1}{2}\|u\|^{2}+\frac{b}{4}\left(\int_{\mathbb{R}^{N}}\left|(-\Delta)^{\frac{\alpha}{2}} u\right|^{2} d x\right)^{2}-\lambda \int_{\mathbb{R}^{N}} F(u) d x .
$$

It is easy to see that $B(u) \geq 0$ for all $u \in \mathcal{H}$ and $A(u) \rightarrow+\infty$ as $\|u\| \rightarrow \infty$.

In the following $H$ denotes a closed half-space of $\mathbb{R}^{N}$ containing the origin, $0 \in H$. We denote by $\mathscr{H}$ the set of closed half-spaces of $\mathbb{R}^{N}$ containing the origin. We shall equip $\mathscr{H}$ with a topology ensuring that $H_{n} \rightarrow H$ as $n \rightarrow \infty$ if there is a sequence of isometries $i_{n}: \mathbb{R}^{N} \rightarrow \mathbb{R}^{N}$ such that $H_{n}=i_{n}(H)$ and $i_{n}$ converges to the identity. Given $x \in \mathbb{R}^{N}$, the reflected point $\sigma_{H}(x)$ will also be denoted by $x^{H}$. The polarization of a nonnegative function $u: \mathbb{R}^{N} \rightarrow \mathbb{R}_{+}$with respect to $H$ is defined as

$$
u^{H}(x):= \begin{cases}\max \left\{u(x), u\left(\sigma_{H}(x)\right)\right\}, & \text { for } x \in H, \\ \min \left\{u(x), u\left(\sigma_{H}(x)\right)\right\}, & \text { for } x \in \mathbb{R}^{N} \backslash H .\end{cases}
$$

Given $u$, the Schwarz symmetrization $u^{*}$ of $u$ is the unique function such that $u$ and $u^{*}$ are equimeasurable and $u^{*}(x)=h(|x|)$, where $h:(0, \infty) \rightarrow \mathbb{R}_{+}$is a continuous and decreasing function.

We set $\mathcal{H}^{+}:=\{u \in \mathcal{H}: u \geq 0\}$. Now we state a symmetric version of Theorem 3.1.

Lemma 3.2 (Symmetric monotonicity trick [32]). Under the assumptions of Theorem 3.1 for $E=\mathcal{H}$, assume that $I_{\lambda}(|u|) \leq I_{\lambda}(u)$ for any $\lambda \in J$ and $u \in \mathcal{H}$ and

$$
I_{\lambda}\left(u^{H}\right) \leq I_{\lambda}(u), \quad \text { for any } \lambda \in J, u \in \mathcal{H}^{+} \text {and } H \in \mathscr{H} \text {. }
$$

Then, for any $p \in\left[2,2_{\alpha}^{*}\right], I_{\lambda}$ has a bounded Palais-Smale sequence $\left\{u_{n}\right\}_{n \in \mathbb{N}} \subset \mathcal{H}$ with $\left\|u_{n}-\left|u_{n}\right|^{*}\right\|_{p} \rightarrow 0$.

Lemma 3.3 (Uniform Mountain-Pass geometry). Assume that $\left(\mathrm{f}_{1}\right)-\left(\mathrm{f}_{3}\right)$ and $\left(\mathrm{V}_{1}\right)-\left(\mathrm{V}_{3}\right)$ hold. Furthermore let $N=2$ with $\alpha \in\left(\frac{1}{2}, 1\right)$ or $N=3$ with $\alpha \in\left(\frac{3}{4}, 1\right)$. Then we have:

(1) $\Gamma_{\lambda} \neq \emptyset$, for every $\lambda \in J$;

(2) there exist $r, \eta>0$ independent of $\lambda$, such that $\|u\|=r$ implies $I_{\lambda}(u) \geq \eta$. In particular $c_{\lambda} \geq \eta$.

Proof. (1) For every $\varphi \in \mathcal{H}^{+} \backslash\{0\}$, taking into account of $\left(\mathrm{f}_{3}\right)$, we have

$$
I_{\lambda}(\varphi) \leq \frac{1}{2}\|\varphi\|^{2}+\frac{b}{4}\left(\int_{\mathbb{R}^{N}}\left|(-\Delta)^{\frac{\alpha}{2}} \varphi\right|^{2} d x\right)^{2}-\frac{D}{2 q} \int_{\mathbb{R}^{N}} \varphi^{q} d x-\frac{1}{22_{\alpha}^{*}} \int_{\mathbb{R}^{N}} \varphi^{2_{\alpha}^{*}} d x .
$$

Under the assumptions on $N$ and $\alpha$, it follows that $2_{\alpha}^{*}>4$. Then there exists $t_{0}>0$ sufficiently large, independent of $\lambda \in J$, such that $I_{\lambda}\left(t_{0} \varphi\right)<0$. Setting $w:=t_{0} \varphi \in \mathcal{H}$, we have $I_{\lambda}(w)<0$ and we can define the corresponding $\Gamma_{\lambda}$. Then, setting $\gamma(t):=t w$, we have $\gamma \in \Gamma_{\lambda} \neq \emptyset$ for every $\lambda \in J$.

(ii) $\left(\mathrm{f}_{1}\right)-\left(\mathrm{f}_{2}\right)$ imply that, for any $\varepsilon>0$, there exists $C_{\varepsilon}>0$ such that

$$
|F(s)| \leq \varepsilon|s|^{2}+C_{\varepsilon}|s|^{2_{\alpha}^{*}}, \quad \text { for all } s \in \mathbb{R} .
$$

Then there exist $\sigma_{1}, \sigma_{2}>0$ such that

$$
I_{\lambda}(\varphi) \geq \sigma_{1}\|\varphi\|^{2}-\sigma_{2}\|\varphi\|^{2_{\alpha}^{*}}, \quad \text { for every } \varphi \in \mathcal{H}
$$

Hence there exist $r, \eta>0$, independent of $\lambda$, such that for $\|u\|=r, I_{\lambda}(u) \geq \eta$ (and $I_{\lambda}(\varphi)>0$ as soon as $\|\varphi\| \leq r$ with $\varphi \neq 0)$. Now fix $\lambda \in J$ and $\gamma \in \Gamma_{\lambda}$. Since $\gamma(0)=0$ and $I_{\lambda}(\gamma(1))<0$, certainly $\|\gamma(1)\|>r$. By continuity, we conclude that there exists $t_{\gamma} \in(0,1)$ such that $\left\|\gamma\left(t_{\gamma}\right)\right\|=r$. Therefore, for every $\lambda \in J$, we conclude $c_{\lambda} \geq \inf _{\gamma \in \Gamma_{\lambda}} I_{\lambda}\left(\gamma\left(t_{\gamma}\right)\right) \geq \eta>0$. 
Lemma 3.4 ( $I_{\lambda}$ decreases upon polarization). Assume $\left(\mathrm{V}_{4}\right)$ holds. Then for any $\lambda \in J$, for all $u \in \mathcal{H}^{+}$and $H \in \mathscr{H}$ there holds $I_{\lambda}\left(u^{H}\right) \leq I_{\lambda}(u)$.

Proof. It is known (see [5, Theorem 2]) that

$$
\int_{\mathbb{R}^{2 N}} \frac{\left|u^{H}(x)-u^{H}(y)\right|^{2}}{|x-y|^{N+2 \alpha}} d x d y \leq \int_{\mathbb{R}^{2 N}} \frac{|u(x)-u(y)|^{2}}{|x-y|^{N+2 \alpha}} d x d y, \quad \text { for all } u \in \mathcal{H}^{+} .
$$

Furthermore, we have (see [34])

$$
\int_{\mathbb{R}^{N}} F\left(u^{H}\right) d x=\int_{\mathbb{R}^{N}} F(u) d x, \quad \text { for all } u \in \mathcal{H}^{+},
$$

and, by the monotonicity assumptions on $V$,

$$
\int_{\mathbb{R}^{N}} V(x)\left(u^{H}\right)^{2} d x \leq \int_{\mathbb{R}^{N}} V(x) u^{2} d x, \quad \text { for all } u \in \mathcal{H}^{+},
$$

which concludes the proof by the definition of $I_{\lambda}$.

Assume $\left(V_{1}\right)-\left(V_{3}\right)$ and $\left(f_{1}\right)-\left(f_{3}\right)$. As a consequence we now get the following result.

Corollary 3.5 (Bounded Palais-Smale with sign). For almost every $\lambda \in J$, there is a bounded sequence $\left\{u_{n}\right\}_{n \in \mathbb{N}} \subset \mathcal{H}^{+}$such that $I_{\lambda}\left(u_{n}\right) \rightarrow c_{\lambda}, I_{\lambda}^{\prime}\left(u_{n}\right) \rightarrow 0$. Furthermore, $\left\|u_{n}-\left|u_{n}\right|^{*}\right\|_{2_{\alpha}^{*}} \rightarrow 0$ if $\left(\mathrm{V}_{4}\right)$ is assumed.

Proof. For a.a. $\lambda \in J$, a bounded (PS)-sequence $\left\{u_{n}\right\}_{n \in \mathbb{N}} \subset \mathcal{H}$ for $I_{\lambda}$ is provided by combining Theorem 3.1 with Lemmas 3.3 and 3.4. Furthermore, if $\left(\mathrm{V}_{4}\right)$ holds, using Lemma 3.2 in place of Theorem 3.1, we also get $\left\|u_{n}-\left|u_{n}\right|^{*}\right\|_{2_{\alpha}^{*}} \rightarrow 0$. Next we show that we can assume that $u_{n}$ is nonnegative. Indeed, we know that $\left\langle I_{\lambda}^{\prime}\left(u_{n}\right), u_{n}^{-}\right\rangle=\left\langle\mu_{n}, u_{n}^{-}\right\rangle$with $\mu_{n} \rightarrow 0$ in $\mathcal{H}^{\prime}$ as $n \rightarrow \infty$, with $u_{n}^{-}=\min \left\{u_{n}, 0\right\}$, namely $(f(s)=0$ for $s \leq 0)$

$$
\left(a+b \int_{\mathbb{R}^{N}}\left|(-\Delta)^{\frac{\alpha}{2}} u_{n}\right|^{2} d x\right) \int_{\mathbb{R}^{N}}(-\Delta)^{\alpha} u_{n} u_{n}^{-} d x+\int_{\mathbb{R}^{N}} V(x)\left|u_{n}^{-}\right|^{2} d x=\left\langle\mu_{n}, u_{n}^{-}\right\rangle .
$$

As it is readily checked, for all $x, y \in \mathbb{R}^{N}$, we have

$$
\left(u_{n}(x)-u_{n}(y)\right)\left(u_{n}^{-}(x)-u_{n}^{-}(y)\right) \geq\left(u_{n}^{-}(x)-u_{n}^{-}(y)\right)^{2},
$$

which yields that

$$
\begin{aligned}
2 C(n, \alpha)^{-1} \int_{\mathbb{R}^{3}}(-\Delta)^{\alpha} u_{n} u_{n}^{-} d x & =\int_{\mathbb{R}^{2 N}} \frac{\left[u_{n}(x)-u_{n}(y)\right]\left[u_{n}^{-}(x)-u_{n}^{-}(y)\right]}{|x-y|^{N+2 \alpha}} d x d y \\
& \geq \int_{\mathbb{R}^{2 N}} \frac{\left[u_{n}^{-}(x)-u_{n}^{-}(y)\right]^{2}}{|x-y|^{N+2 \alpha}} d x d y=2 C(n, \alpha)^{-1}\left\|u_{n}^{-}\right\|_{D^{\alpha, 2}}^{2} .
\end{aligned}
$$

Thus $\left\|u_{n}^{-}\right\|=o_{n}(1)$, which also yields that $\left\{u_{n}^{+}\right\}_{n \in \mathbb{N}}$ is bounded. We can now prove that $I_{\lambda}\left(u_{n}^{+}\right) \rightarrow c_{\lambda}$ and $I_{\lambda}^{\prime}\left(u_{n}^{+}\right) \rightarrow 0$. Of course $\left\|u_{n}\right\|^{2}=\left\|u_{n}^{+}\right\|^{2}+o_{n}(1)$ and

$$
\left(\int_{\mathbb{R}^{N}}\left|(-\Delta)^{\frac{\alpha}{2}} u_{n}\right|^{2} d x\right)^{2}=\left(\int_{\mathbb{R}^{N}}\left|(-\Delta)^{\frac{\alpha}{2}} u_{n}^{+}\right|^{2} d x\right)^{2}+o_{n}(1) .
$$

Notice that from $\left(f_{1}\right)-\left(f_{2}\right)$, we get

$$
\left|\int_{\mathbb{R}^{N}} F\left(u_{n}\right) d x-\int_{\mathbb{R}^{N}} F\left(u_{n}^{+}\right) d x\right| \leq C \int_{\mathbb{R}^{N}}\left(\left|u_{n}\right|+\left|u_{n}\right|^{2_{\alpha}^{*}-1}\right)\left|u_{n}^{-}\right| \leq C\left\|u_{n}^{-}\right\|_{2}+C\left\|u_{n}^{-}\right\|_{2_{\alpha}^{*}}=o_{n}(1) .
$$


This shows that $I_{\lambda}\left(u_{n}^{+}\right) \rightarrow c_{\lambda}$. We claim that $I_{\lambda}^{\prime}\left(u_{n}^{+}\right) \rightarrow 0$. Setting $w_{n}:=I_{\lambda}^{\prime}\left(u_{n}\right)-I_{\lambda}^{\prime}\left(u_{n}^{+}\right)$, it is enough to prove that $w_{n} \rightarrow 0$ in $\mathcal{H}^{\prime}$. For any $\varphi \in \mathcal{H}$ with $\|\varphi\|_{\mathcal{H}} \leq 1$, we have

$$
\begin{aligned}
\left\langle w_{n}, \varphi\right\rangle & =\left(a+b \int_{\mathbb{R}^{N}}\left|(-\Delta)^{\frac{\alpha}{2}} u_{n}\right|^{2} d x\right) \int_{\mathbb{R}^{N}}(-\Delta)^{\alpha / 2} u_{n}(-\Delta)^{\alpha / 2} \varphi d x \\
& -\left(a+b \int_{\mathbb{R}^{N}}\left|(-\Delta)^{\frac{\alpha}{2}} u_{n}^{+}\right|^{2} d x\right) \int_{\mathbb{R}^{N}}(-\Delta)^{\alpha / 2} u_{n}^{+}(-\Delta)^{\alpha / 2} \varphi d x \\
& +\int_{\mathbb{R}^{N}} V(x) u_{n}^{-} \varphi d x-\lambda \int_{\mathbb{R}^{N}}\left(f\left(u_{n}\right)-f\left(u_{n}^{+}\right)\right) \varphi d x \\
& =\left(a+b \int_{\mathbb{R}^{N}}\left|(-\Delta)^{\frac{\alpha}{2}} u_{n}^{+}\right|^{2} d x\right) \int_{\mathbb{R}^{N}}(-\Delta)^{\alpha / 2} u_{n}^{-}(-\Delta)^{\alpha / 2} \varphi d x \\
& +\int_{\mathbb{R}^{N}} V(x) u_{n}^{-} \varphi d x-\lambda \int_{\mathbb{R}^{N}} f\left(u_{n}^{-}\right) \varphi d x+\left\langle\xi_{n}, \varphi\right\rangle,
\end{aligned}
$$

for some $\xi_{n} \rightarrow 0$ in $\mathcal{H}^{\prime}$. Then, using $\left(f_{1}\right)-\left(f_{2}\right)$ again, $\left|\left\langle w_{n}, \varphi\right\rangle\right| \leq C\left\|u_{n}^{-}\right\|_{\mathcal{H}}+\left\|\xi_{n}\right\|_{\mathcal{H}^{\prime}}$, proving the claim. Observe now that by the triangular inequality and the contractility property of the Schwarz symmetrization in $L^{p}$-spaces (i.e. $\left\|w^{*}-z^{*}\right\|_{p} \leq\|w-z\|_{p}$ for all $w, z \in L^{p}\left(\mathbb{R}^{N}\right)$ with $w, z \geq 0$ ), we get

$$
\begin{aligned}
& \left|\left\|u_{n}^{+}-\left(u_{n}^{+}\right)^{*}\right\|_{2_{\alpha}^{*}}-\left\|u_{n}-\left|u_{n}\right|^{*}\right\|_{2_{\alpha}^{*}}\right| \\
& \leq\left\|u_{n}^{-}+\left(\left(u_{n}^{+}\right)^{*}-\left|u_{n}\right|^{*}\right)\right\|_{2_{\alpha}^{*}} \leq\left\|u_{n}^{-}\right\|_{2_{\alpha}^{*}}+\left\|\left(u_{n}^{+}\right)^{*}-\left|u_{n}\right|^{*}\right\|_{2_{\alpha}^{*}} \\
& \leq\left\|u_{n}^{-}\right\|_{2_{\alpha}^{*}}+\left\|u_{n}^{+}-\left|u_{n}\right|\right\|_{2_{\alpha}^{*}}=2\left\|u_{n}^{-}\right\|_{2_{\alpha}^{*}} \leq C\left\|u_{n}^{-}\right\|_{\mathcal{H}}=o_{n}(1) .
\end{aligned}
$$

Since $\left\|u_{n}-\left|u_{n}\right|^{*}\right\|_{2_{\alpha}^{*}} \rightarrow 0$, we have $\left\|u_{n}^{+}-\left(u_{n}^{+}\right)^{*}\right\|_{2_{\alpha}^{*}} \rightarrow 0$ as $n \rightarrow \infty$. This ends the proof.

\section{UPPER ESTIMATE OF $c_{\lambda}$ AND LIMIT PROBLEMS}

In this section, we give an upper estimate of the mountain pass value $c_{\lambda}$. Moreover, the corresponding limit problem is discussed.

4.1. An energy estimate. Next we provide a crucial energy estimate for $c_{\lambda}$.

Lemma 4.1 (Energy estimate). Suppose that $\left(\mathrm{f}_{1}\right)-\left(\mathrm{f}_{3}\right)$ and $\left(\mathrm{V}_{1}\right)-\left(\mathrm{V}_{3}\right)$ hold. For any $\lambda \in\left[\frac{1}{2}, 1\right]$, assume that

Then we have

$$
q \in\left(\frac{4 \alpha}{N-2 \alpha}, 2_{\alpha}^{*}\right) \quad \text { or } \quad q \in\left(2, \frac{4 \alpha}{N-2 \alpha}\right] \text { with D large enough. }
$$

$$
c_{\lambda}<c_{\lambda}^{*}, \quad c_{\lambda}^{*}:=\frac{a S_{\alpha}}{2} T^{N-2 \alpha}+\frac{b S_{\alpha}^{2}}{4} T^{2 N-4 \alpha}-\frac{\lambda}{2_{\alpha}^{*}} T^{N},
$$

where $T=T(\lambda)>0$ continuously depends on $\lambda$.

Proof. Let $\eta \in C_{0}^{\infty}\left(\mathbb{R}^{3}\right)$ be a cut-off function with support in $B_{2}(0)$ such that $\eta \equiv 1$ on $B_{1}(0)$ and $\eta \in[0,1]$ on $B_{2}(0)$. It is well known that $S_{\alpha}$ is achieved by

$$
\mathcal{T}(x):=\kappa\left(\mu^{2}+\left|x-x_{0}\right|^{2}\right)^{-\frac{N-2 \alpha}{2}}
$$

for arbitrary $\kappa \in \mathbb{R}, \mu>0$ and $x_{0} \in \mathbb{R}^{N}$. Then, taking $x_{0}=0$, we can define

$$
v_{\varepsilon}(x):=\eta(x) u_{\varepsilon}(x), \quad u_{\varepsilon}(x)=\varepsilon^{-\frac{N-2 \alpha}{2}} u^{*}(x / \varepsilon), \quad u^{*}(x):=\frac{\mathcal{T}\left(x / S_{\alpha}^{1 /(2 \alpha)}\right)}{\|\mathcal{T}\|_{2_{\alpha}^{*}}}
$$


Then $(-\Delta)^{\alpha} u_{\varepsilon}=\left|u_{\varepsilon}\right|^{2_{\alpha}^{*}-2} u_{\varepsilon}$ and $\left\|(-\Delta)^{\frac{\alpha}{2}} u_{\varepsilon}\right\|_{2}^{2}=\left\|u_{\varepsilon}\right\|_{2_{\alpha}^{*}}^{2_{\alpha}^{*}}=S_{\alpha}^{\frac{N}{2 \alpha}}$. As in [30], we have

$$
A_{\varepsilon}:=\int_{\mathbb{R}^{N}}\left|(-\Delta)^{\frac{\alpha}{2}} v_{\varepsilon}(x)\right|^{2} d x=S_{\alpha}^{\frac{N}{2 \alpha}}+\mathcal{O}\left(\varepsilon^{N-2 \alpha}\right) .
$$

On the other hand, for any $q \in\left[2,2_{\alpha}^{*}\right)$, we obtain

$$
\int_{\mathbb{R}^{N}}\left|v_{\varepsilon}\right|^{q} d x \geq \varepsilon^{N-\frac{(N-2 \alpha) q}{2}} \kappa^{q}\|\mathcal{T}\|_{2_{\alpha}^{*}}^{-q}\left|\mathbb{S}_{N-1}\right| S_{\alpha}^{N /(2 \alpha)} \int_{0}^{\frac{1}{\varepsilon S_{\alpha}^{1 /(2 \alpha)}}} \frac{r^{N-1}}{\left(\mu^{2}+r^{2}\right)^{\frac{(N-2 \alpha) q}{2}}} d r,
$$

where $\mathbb{S}_{N-1}$ is the unit sphere in $\mathbb{R}^{N}$. Observe that, as $\varepsilon \rightarrow 0$,

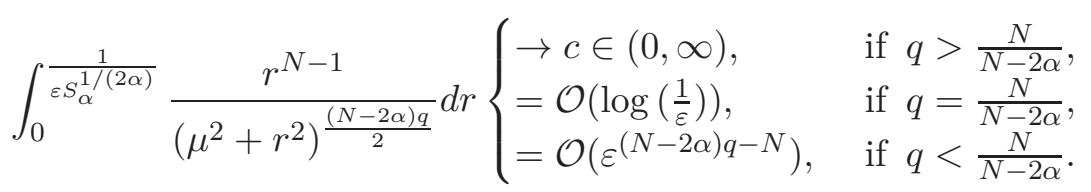

Then

$$
C_{\varepsilon}:=\int_{\mathbb{R}^{N}}\left|v_{\varepsilon}\right|^{q} d x \geq \begin{cases}\mathcal{O}\left(\varepsilon^{\left.N-\frac{(N-2 \alpha) q}{2}\right),}\right. & \text { if } q>\frac{N}{N-2 \alpha} \\ \mathcal{O}\left(\log \left(\frac{1}{\varepsilon}\right) \varepsilon^{N-\frac{(N-2 \alpha) q}{2}}\right), & \text { if } q=\frac{N}{N-2 \alpha} \\ \mathcal{O}\left(\varepsilon^{\frac{(N-2 \alpha) q}{2}}\right), & \text { if } q<\frac{N}{N-2 \alpha} .\end{cases}
$$

Since $2<\frac{N}{N-2 \alpha}$,

$$
\int_{\mathbb{R}^{N}}\left|v_{\varepsilon}\right|^{2} d x \geq \mathcal{O}\left(\varepsilon^{N-2 \alpha}\right)
$$

Similar as above,

$$
\int_{\mathbb{R}^{N}}\left|v_{\varepsilon}\right|^{2} d x \leq \varepsilon^{2 \alpha} \kappa^{2}\|\mathcal{T}\|_{2_{\alpha}^{*}}^{-2}\left|\mathbb{S}_{N-1}\right| S_{\alpha}^{N /(2 \alpha)} \int_{0}^{\frac{2}{\varepsilon S_{\alpha}^{1 /(2 \alpha)}}} \frac{r^{N-1}}{\left(\mu^{2}+r^{2}\right)^{N-2 \alpha}} d r \leq \mathcal{O}\left(\varepsilon^{N-2 \alpha}\right) .
$$

So that we have

$$
B_{\varepsilon}:=\int_{\mathbb{R}^{N}}\left|v_{\varepsilon}\right|^{2} d x=\mathcal{O}\left(\varepsilon^{N-2 \alpha}\right) .
$$

As can be seen in [30], it holds

$$
D_{\varepsilon}:=\int_{\mathbb{R}^{N}}\left|v_{\varepsilon}\right|^{2_{\alpha}^{*}} d x=S_{\alpha}^{\frac{N}{2 \alpha}}+\mathcal{O}\left(\varepsilon^{N}\right) .
$$

Step 1. For any $\varepsilon>0$ small there exists $t_{0}>0$ such that $I_{\lambda}\left(\gamma_{\varepsilon}\left(t_{0}\right)\right)<0$, where $\gamma_{\varepsilon}(t):=v_{\varepsilon}(\cdot / t)$. Indeed, by $\left(\mathrm{V}_{2}\right)$ and $\left(\mathrm{f}_{3}\right)$, for any $t>0$,

$$
\begin{aligned}
I_{\lambda}\left(\gamma_{\varepsilon}(t)\right) \leq & \frac{a}{2} \int_{\mathbb{R}^{N}}\left|(-\Delta)^{\frac{\alpha}{2}} \gamma_{\varepsilon}(t)\right|^{2} d x+\frac{b}{4}\left(\int_{\mathbb{R}^{N}}\left|(-\Delta)^{\frac{\alpha}{2}} \gamma_{\varepsilon}(t)\right|^{2} d x\right)^{2} \\
& +\frac{V_{\infty}}{2} \int_{\mathbb{R}^{N}}\left|\gamma_{\varepsilon}(t)\right|^{2} d x-\lambda \int_{\mathbb{R}^{N}}\left[\frac{\left|\gamma_{\varepsilon}(t)\right|^{2_{\alpha}^{*}}}{2_{\alpha}^{*}}+D \frac{\left|\gamma_{\varepsilon}(t)\right|^{q}}{q}\right] d x \\
= & \frac{a A_{\varepsilon}}{2} t^{N-2 \alpha}+\frac{b A_{\varepsilon}^{2}}{4} t^{2 N-4 \alpha}+\left(\frac{V_{\infty} B_{\varepsilon}}{2}-\frac{\lambda D_{\varepsilon}}{2_{\alpha}^{*}}-\frac{\lambda D C_{\varepsilon}}{q}\right) t^{N} .
\end{aligned}
$$

Noting that $2 \alpha<N<4 \alpha$, we have $0<2 N-4 \alpha<N$. Then by (4.3),

$$
\frac{V_{\infty} B_{\varepsilon}}{2}-\frac{\lambda D_{\varepsilon}}{2_{\alpha}^{*}} \rightarrow-\frac{\lambda S_{\alpha}^{\frac{N}{2 \alpha}}}{2_{\alpha}^{*}}, \text { as } \varepsilon \rightarrow 0 .
$$


So it follows from (4.1) that for any $\varepsilon>0$ small enough, $I_{\lambda}\left(\gamma_{\varepsilon}(t) \rightarrow-\infty\right.$ as $t \rightarrow+\infty$. Then there exists $t_{0}>0$ such that $I_{\lambda}\left(\gamma_{\varepsilon}\left(t_{0}\right)\right)<0$.

Step 2. Notice that, as $t \rightarrow 0^{+}$, we have

$$
\int_{\mathbb{R}^{N}}\left[\left|(-\Delta)^{\frac{\alpha}{2}} \gamma_{\varepsilon}(t)\right|^{2}+\left|\gamma_{\varepsilon}(t)\right|^{2}\right] d x=t^{N-2 \alpha} A_{\varepsilon}+t^{N} B_{\varepsilon} \rightarrow 0
$$

uniformly for $\varepsilon>0$ small. We set $\gamma_{\varepsilon}(0)=0$. Then $\gamma_{\varepsilon}\left(t_{0} \cdot\right) \in \Gamma_{\lambda}$, where $\Gamma_{\lambda}$ is as in Theorem 3.1 and

$$
c_{\lambda} \leq \sup _{t \geq 0} I_{\lambda}\left(\gamma_{\varepsilon}(t)\right)
$$

Recalling that $c_{\lambda}>0$, by (4.4), there exists $t_{\varepsilon}>0$ such that

$$
\sup _{t \geq 0} I_{\lambda}\left(\gamma_{\varepsilon}(t)\right)=I_{\lambda}\left(\gamma_{\varepsilon}\left(t_{\varepsilon}\right)\right)
$$

By (4.1), (4.2) and (4.4), we get $I_{\lambda}\left(\gamma_{\varepsilon}(t)\right) \rightarrow 0^{+}$as $t \rightarrow 0^{+}$and $I_{\lambda}\left(\gamma_{\varepsilon}(t)\right) \rightarrow-\infty$ as $t \rightarrow+\infty$ uniformly for $\varepsilon>0$ small. Then there exist $t_{1}, t_{2}>0$ (independent of $\varepsilon>0$ ) such that $t_{1} \leq t_{\varepsilon} \leq t_{2}$. Let

then

$$
J_{\varepsilon}(t):=\frac{a A_{\varepsilon}}{2} t^{N-2 \alpha}+\frac{b A_{\varepsilon}^{2}}{4} t^{2 N-4 \alpha}-\frac{\lambda D_{\varepsilon}}{2_{\alpha}^{*}} t^{N}
$$

$$
c_{\lambda} \leq \sup _{t \geq 0} J_{\varepsilon}(t)+\left(\frac{V_{\infty} B_{\varepsilon}}{2}-\frac{\lambda D C_{\varepsilon}}{q}\right) t_{\varepsilon}^{N}
$$

By formula (4.2), for any $q \in\left(2,2_{\alpha}^{*}\right)$, we have

$$
C_{\varepsilon} \geq \mathcal{O}\left(\varepsilon^{N-\frac{(N-2 \alpha) q}{2}}\right) .
$$

Then by (4.3), we conclude that

$$
c_{\lambda} \leq \sup _{t \geq 0} J_{\varepsilon}(t)+\mathcal{O}\left(\varepsilon^{N-2 \alpha}\right)-\mathcal{O}\left(D \varepsilon^{N-\frac{(N-2 \alpha) q}{2}}\right) .
$$

Noting that $N-2 \alpha>0$ and $N-(N-2 \alpha) q / 2>0$, we have $\sup _{t \geq 0} J_{\varepsilon}(t) \geq c_{\lambda} / 2$ uniformly for $\varepsilon>0$ small. As above, there are $t_{3}, t_{4}>0$ (independent of $\varepsilon>0$ ) such that $\sup _{t \geq 0} J_{\varepsilon}(t)=$ $\sup _{t \in\left[t_{3}, t_{4}\right]} J_{\varepsilon}(t)$. By (4.1),

$$
c_{\lambda} \leq \sup _{t \geq 0} K\left(S_{\alpha}^{\frac{1}{2 \alpha}} t\right)+\mathcal{O}\left(\varepsilon^{N-2 \alpha}\right)-\mathcal{O}\left(D \varepsilon^{N-\frac{(N-2 \alpha) q}{2}}\right),
$$

where

$$
K(t)=\frac{a S_{\alpha}}{2} t^{N-2 \alpha}+\frac{b S_{\alpha}^{2}}{4} t^{2 N-4 \alpha}-\frac{\lambda}{2_{\alpha}^{*}} t^{N}
$$

Observe that for $t>0$,

$$
K^{\prime}(t)=\frac{(N-2 \alpha) t^{N-2 \alpha-1}}{2} \tilde{K}(t), \text { where } \tilde{K}(t):=a S_{\alpha}+b S_{\alpha}^{2} t^{N-2 \alpha}-\lambda t^{2 \alpha},
$$

and $\tilde{K}^{\prime}(t)=t^{N-2 \alpha-1}\left(b S_{\alpha}^{2}(N-2 \alpha)-2 \lambda \alpha t^{4 \alpha-N}\right)$. Since $4 \alpha>N$, there is a unique $T>0$ such that $\tilde{K}(t)>0$ if $t \in(0, T)$ and $\tilde{K}(t)<0$ if $t>T$. Hence, $T$ is the unique maximum point of $K$. Then by (4.5),

$$
c_{\lambda} \leq K(T)+\mathcal{O}\left(\varepsilon^{N-2 \alpha}\right)-\mathcal{O}\left(D \varepsilon^{N-\frac{(N-2 \alpha) q}{2}}\right) .
$$


If $q>4 \alpha /(N-2 \alpha)$, then $0<N-(N-2 \alpha) q / 2<N-2 \alpha$, which implies by (4.6) that for any fixed $D>0, c_{\lambda}<K(T)$ for $\varepsilon>0$ small. If $2<q \leq 4 \alpha /(N-2 \alpha)$, for $\varepsilon>0$ small and $D \geq \varepsilon^{(N-2 \alpha) q / 2-2 \alpha-1}$, then also in this case $c_{\lambda}<K(T)$, which completes the proof.

4.2. The limit problem. Note that $V(x) \rightarrow V_{\infty}$ as $|x| \rightarrow \infty$. For any $\lambda \in[1 / 2,1]$, we consider the problem

$$
\left\{\begin{array}{r}
\left(a+b \int_{\mathbb{R}^{N}}\left|(-\Delta)^{\frac{\alpha}{2}} u\right|^{2} d x\right)(-\Delta)^{\alpha} u+V_{\infty} u=\lambda f(u) \quad \text { in } \mathbb{R}^{N}, \\
u \in H^{\alpha}\left(\mathbb{R}^{N}\right), \quad u>0 \text { in } \mathbb{R}^{N},
\end{array}\right.
$$

whose energy functional is defined by

$$
I_{\lambda}^{\infty}(u):=\frac{1}{2} \int_{\mathbb{R}^{N}}\left(a\left|(-\Delta)^{\frac{\alpha}{2}} u\right|^{2}+V_{\infty} u^{2}\right) d x+\frac{b}{4}\left(\int_{\mathbb{R}^{N}}\left|(-\Delta)^{\frac{\alpha}{2}} u\right|^{2} d x\right)^{2}-\lambda \int_{\mathbb{R}^{N}} F(u) d x .
$$

We will use of the following Pohožaev type identity, whose proof is similar as in [10].

Lemma 4.2 (Pohožaev identity). Let $u$ be a critical point of $I_{\lambda}^{\infty}$ in $\mathcal{H}$ for $\lambda \in\left[\frac{1}{2}, 1\right]$. Then $P_{\lambda}(u)=0$,

$$
\begin{aligned}
P_{\lambda}(u):= & \frac{N-2 \alpha}{2} \int_{\mathbb{R}^{N}} a\left|(-\Delta)^{\frac{\alpha}{2}} u\right|^{2} d x+\frac{N-2 \alpha}{2} b\left(\int_{\mathbb{R}^{N}}\left|(-\Delta)^{\frac{\alpha}{2}} u\right|^{2} d x\right)^{2} \\
& +\frac{N}{2} \int_{\mathbb{R}^{N}} V_{\infty} u^{2} d x-N \lambda \int_{\mathbb{R}^{N}} F(u) d x .
\end{aligned}
$$

Notice that $P_{\lambda}(u)=\left.\frac{d}{d t} I_{\lambda}^{\infty}(u(\cdot / t))\right|_{t=1}$.

Lemma 4.3. For $\lambda \in\left[\frac{1}{2}, 1\right]$, if $w_{\lambda} \in \mathcal{H} \backslash\{0\}$ solves $P_{\lambda}\left(w_{\lambda}\right)=0$, then there exists $\gamma_{\lambda} \in C([0,1], \mathcal{H})$ such that $\gamma_{\lambda}(0)=0, I_{\lambda}^{\infty}\left(\gamma_{\lambda}(1)\right)<0, w_{\lambda} \in \gamma_{\lambda}([0,1]), 0 \notin \gamma_{\lambda}((0,1])$ and

$$
\max _{t \in[0,1]} I_{\lambda}^{\infty}\left(\gamma_{\lambda}(t)\right)=I_{\lambda}^{\infty}\left(w_{\lambda}\right)
$$

Proof. Note that

$$
\begin{aligned}
I_{\lambda}^{\infty}\left(w_{\lambda}(\cdot / t)\right)= & \frac{t^{N-2 \alpha}}{2} \int_{\mathbb{R}^{N}} a\left|(-\Delta)^{\frac{\alpha}{2}} w_{\lambda}\right|^{2} d x+\frac{b t^{2 N-4 \alpha}}{4}\left(\int_{\mathbb{R}^{N}}\left|(-\Delta)^{\frac{\alpha}{2}} w_{\lambda}\right|^{2} d x\right)^{2} \\
& +\frac{t^{N}}{2} \int_{\mathbb{R}^{N}} V_{\infty} w_{\lambda}^{2} d x-t^{N} \lambda \int_{\mathbb{R}^{N}} F\left(w_{\lambda}\right) d x=0,
\end{aligned}
$$

which, by (4.7), yields

$$
\lim _{t \rightarrow \infty} I_{\lambda}^{\infty}\left(w_{\lambda}(\cdot / t)\right)<0
$$

Then there is $t_{0}>0$ such that $I_{\lambda}^{\infty}\left(w_{\lambda}\left(\cdot / t_{0}\right)\right)<0$. Let $\left.\gamma_{\lambda}(t)=w_{\lambda}\left(\cdot / t t_{0}\right)\right)$ for $0<t \leq 1$ and $\gamma_{\lambda}(0)=0$. Then $\gamma_{\lambda} \in C([0,1], \mathcal{H}), w_{\lambda} \in \gamma_{\lambda}([0,1])$ and $\max _{t \in[0,1]} I_{\lambda}^{\infty}\left(\gamma_{\lambda}(t)\right)=I_{\lambda}^{\infty}\left(w_{\lambda}\right)$ as $t=t_{0}^{-1}$ is the unique maximum point of $t \mapsto I_{\lambda}^{\infty}\left(\gamma_{\lambda}(t)\right)$ by Lemma 4.2 .

\section{Behaviour of Palais-Smale sequences}

By Corollary 3.5, for almost every $\lambda \in[1 / 2,1]$, there exists a bounded Palais-Smale sequence $\left\{u_{n}\right\}_{n \in \mathbb{N}} \subset \mathcal{H}$ for $I_{\lambda}$ at the level $c_{\lambda}$. Then there exists a subsequence of $\left\{u_{n}\right\}_{n \in \mathbb{N}}$, still denoted by $\left\{u_{n}\right\}_{n \in \mathbb{N}}$, such that $u_{n} \rightarrow u_{0}$ in $\mathcal{H}$ and $u_{n} \rightarrow u_{0}$ a.e. in $\mathbb{R}^{N}$ as $n \rightarrow \infty$. Let $v_{n}^{1}:=u_{n}-u_{0}$, then $v_{n}^{1} \rightarrow 0$ in $\mathcal{H}$ and $v_{n}^{1} \rightarrow 0$ a.e. in $\mathbb{R}^{N}$. 
5.1. Splitting lemmas. Let us set

$$
g(t):=f(t)-\left(t^{+}\right)^{2_{\alpha}^{*}-1}, \quad G(t):=\int_{0}^{t} g(s) d s .
$$

In order to get the profile decomposition of $\left\{u_{n}\right\}_{n \in \mathbb{N}}$, we state the following splitting lemmas.

Lemma 5.1 (Splitting lemma I). We have

$$
\left|\int_{\mathbb{R}^{N}}\left(g\left(u_{n}\right)-g\left(u_{0}\right)-g\left(v_{n}^{1}\right)\right) \varphi d x\right| \leq o_{n}(1)\|\varphi\|,
$$

where $o_{n}(1) \rightarrow 0$ as $n \rightarrow \infty$, uniformly for any $\varphi \in C_{0}^{\infty}\left(\mathbb{R}^{N}\right)$.

Proof. For each $n \geq 1$, there exists $\theta_{n} \in(0,1)$ such that

$$
\left|g\left(u_{n}\right)-g\left(v_{n}^{1}\right)\right| \leq\left|g^{\prime}\left(v_{n}^{1}+\theta_{n} u_{0}\right)\right|\left|u_{0}\right| .
$$

In view of $\left(\mathrm{f}_{1}\right)-\left(\mathrm{f}_{3}\right)$, for any $\varepsilon>0$, there exists $\bar{D}>0$ such that

$$
|g(t)| \leq \varepsilon|t|^{2_{\alpha}^{*}-1}, \quad \text { for }|t| \geq \bar{D} / 2 .
$$

Let $\Omega_{n}(\bar{D}):=\left\{x \in \mathbb{R}^{N}:\left|u_{n}(x)\right| \geq \bar{D}\right\}$ and for $r>0, B_{r}:=\left\{x \in \mathbb{R}^{N}:|x|<r\right\}, B_{r}^{c}:=\mathbb{R}^{N} \backslash B_{r}(0)$. Since $u_{0} \in \mathcal{H}$, we have $\left|B_{R}^{c} \cap\left\{\left|u_{0}(x)\right| \geq \bar{D} / 2\right\}\right| \rightarrow 0$ as $R \rightarrow \infty$. Then for $\varepsilon$ given as above, there exist $R>0$ and $\Omega_{R} \subset \mathbb{R}^{N^{N}}$ with $\left|\Omega_{R}\right| \leq \Lambda_{\varepsilon}$ such that $\left|u_{0}(x)\right|<\bar{D} / 2$ for $x \in B_{R}^{c} \backslash \Omega_{R}$, where $\Lambda_{\varepsilon}>0$ will be chosen later small enough. Then, by Hölder's inequality, (5.2) and (5.3), we have

$$
\begin{aligned}
& \int_{B_{R}^{c} \backslash \Omega_{R}}\left|g\left(u_{n}\right)-g\left(v_{n}^{1}\right) \| \varphi\right| d x \\
& \leq \int_{\left(B_{R}^{c} \backslash \Omega_{R}\right) \cap \Omega_{n}(\bar{D})}\left|g\left(u_{n}\right)-g\left(v_{n}^{1}\right)\left\|\varphi\left|d x+\int_{\left(B_{R}^{c} \backslash \Omega_{R}\right) \cap \Omega_{n}^{c}(\bar{D})}\right| g\left(u_{n}\right)-g\left(v_{n}^{1}\right)\right\| \varphi\right| d x \\
& \leq \varepsilon C\left(\left\|u_{n}\right\|_{2_{\alpha}^{*}}^{2^{*}-1}+\left\|v_{n}^{1}\right\|_{2_{\alpha}^{*}}^{2^{*}-1}\right)\|\varphi\|+\max _{|t| \leq 2 \bar{D}}\left|g^{\prime}(t)\right|\left(\int_{B_{R}^{c}} u_{0}^{2}(x) d x\right)^{1 / 2}\|\varphi\| .
\end{aligned}
$$

It follows from $\left(\mathrm{f}_{1}\right)$ and $\left(\mathrm{f}_{2}\right)$ that, for $\varepsilon>0$ given, there exists $C_{\varepsilon}=C_{\varepsilon}(f)>0$ such that

$$
\begin{aligned}
& \int_{\Omega_{R}}\left|g\left(u_{n}\right)-g\left(v_{n}^{1}\right)\right||\varphi| d x \\
& \leq \varepsilon \int_{\Omega_{R}}\left(\left|u_{n}\right|^{2_{\alpha}^{*}-1}+\left|v_{n}^{1}\right|^{2_{\alpha}^{*}-1}\right)|\varphi| d x+C_{\varepsilon} \int_{\Omega_{R}}\left(\left|u_{n}\right|+\left|v_{n}^{1}\right|\right)|\varphi| d x \\
& \leq \varepsilon C\left(\left\|u_{n}\right\|_{2_{\alpha}^{*}}^{2^{*}-1}+\left\|v_{n}^{1}\right\|_{2_{\alpha}^{*}}^{2^{*}-1}\right)\|\varphi\|+C_{\varepsilon}\left|\Omega_{R}\right|^{\frac{2 \alpha}{N}}\left(\left\|u_{n}\right\|_{2_{\alpha}^{*}}+\left\|v_{n}^{1}\right\|_{2_{\alpha}^{*}}\right)\|\varphi\|_{2_{\alpha}^{*}} .
\end{aligned}
$$

By (5.4) and (5.5), by choosing $\Lambda_{\varepsilon}$ such that $C_{\varepsilon} \Lambda_{\varepsilon}^{2 \alpha / N} \leq \varepsilon$, there exists $C>0$ with

$$
\left.\int_{B_{R}^{c}} \mid g\left(u_{n}\right)-g\left(v_{n}^{1}\right)\right)\|\varphi \mid d x \leq C \varepsilon\| \varphi \| .
$$

Moreover,

$$
\begin{aligned}
\int_{B_{R}^{c}}\left|g\left(u_{0}\right) \| \varphi\right| d x & \leq C \int_{B_{R}^{c}}\left|u_{0}\right||\varphi| d x+\int_{B_{R}^{c}}\left|u_{0}\right|^{2_{\alpha}^{*}-1}|\varphi| d x \\
& \leq C\left(\int_{B_{R}^{c}}\left|u_{0}\right|^{2} d x\right)^{1 / 2}\|\varphi\|+C\left(\int_{B_{R}^{c}}\left|u_{0}\right|^{2_{\alpha}^{*}} d x\right)^{\left(2_{\alpha}^{*}-1\right) / 2_{\alpha}^{*}}\|\varphi\| .
\end{aligned}
$$


It follows from (5.6) and (5.7) that, for $\varepsilon>0$ above, we choose $R>0$ above large enough such that

$$
\left|\int_{B_{R}^{c}}\left(g\left(u_{n}\right)-g\left(u_{0}\right)-g\left(v_{n}^{1}\right)\right) \varphi d x\right| \leq C \varepsilon\|\varphi\|,
$$

where $C$ is independent of $n, \varepsilon$ and $\varphi \in C_{0}^{\infty}\left(\mathbb{R}^{N}\right)$. On the other hand,

$$
\int_{B_{R}}\left|g\left(u_{n}\right)-g\left(u_{0}\right)\right||\varphi| d x \leq\left(\int_{B_{R}}\left|g\left(u_{n}\right)-g\left(u_{0}\right)\right|^{2_{\alpha}^{*} /\left(2_{\alpha}^{*}-1\right)} d x\right)^{\left(2_{\alpha}^{*}-1\right) / 2_{\alpha}^{*}}\left(\int_{B_{R}}|\varphi|^{2_{\alpha}^{*}}\right)^{1 / 2_{\alpha}^{*}} .
$$

Observe that

$$
\lim _{t \rightarrow+\infty} \frac{g^{2_{\alpha}^{*} /\left(2_{\alpha}^{*}-1\right)}(t)}{t^{2_{\alpha}^{*}}}=\lim _{t \rightarrow 0^{+}} \frac{g^{2_{\alpha}^{*} /\left(2_{\alpha}^{*}-1\right)}(t)}{t^{2_{\alpha}^{*} /\left(2_{\alpha}^{*}-1\right)}}=0 .
$$

Then $\left|g\left(u_{n}\right)-g\left(u_{0}\right)\right|^{2_{\alpha}^{*} /\left(2_{\alpha}^{*}-1\right)} \rightarrow 0$ in $L^{1}\left(B_{R}\right)$. Hence, we deduce

$$
\int_{B_{R}}\left|g\left(u_{n}\right)-g\left(u_{0}\right)\left\|\varphi \mid d x \leq o_{n}(1)\right\| \varphi \| .\right.
$$

Similarly, we also obtain that

$$
\int_{B_{R}}\left|g\left(v_{n}^{1}\right)\right| \varphi d x \leq o_{n}(1)\|\varphi\|,
$$

for any $\varphi \in C_{0}^{\infty}\left(\mathbb{R}^{N}\right)$. It follows from (5.8), (5.9) and (5.10) that (5.1) holds.

Lemma 5.2 (Splitting lemma II). We have

$$
\left|\int_{\mathbb{R}^{N}}\left(\left|u_{n}\right|^{2_{\alpha}^{*}-2} u_{n}-\left|u_{0}\right|^{2_{\alpha}^{*}-2} u_{0}-\left|v_{n}^{1}\right|^{2_{\alpha}^{*}-2} v_{n}^{1}\right) \varphi d x\right| \leq o_{n}(1)\|\varphi\|,
$$

where $o_{n}(1) \rightarrow 0$ as $n \rightarrow \infty$, uniformly for any $\varphi \in C_{0}^{\infty}\left(\mathbb{R}^{N}\right)$.

Proof. For any $\varepsilon>0$, there exists $R=R(\varepsilon)>0$ such that

$$
\begin{aligned}
& \left|\int_{\mathbb{R}^{N} \backslash B_{R}(0)}\left(\left|u_{n}\right|^{2_{\alpha}^{*}-2} u_{n}-\left|u_{0}\right|^{2_{\alpha}^{*}-2} u_{0}-\left|v_{n}^{1}\right|^{2_{\alpha}^{*}-2} v_{n}^{1}\right) \varphi d x\right| \\
& \leq\left|\int_{\mathbb{R}^{N} \backslash B_{R}(0)}\left(\left|u_{n}\right|^{2_{\alpha}^{*}-2} u_{n}-\left|v_{n}^{1}\right|^{2_{\alpha}^{*}-2} v_{n}^{1}\right) \varphi d x\right|+\left.\left|\int_{\mathbb{R}^{N} \backslash B_{R}(0)}\right| u_{0}\right|^{2_{\alpha}^{*}-2} u_{0} \varphi d x \mid \\
& \leq C \int_{\mathbb{R}^{N} \backslash B_{R}(0)}\left(\left|u_{n}\right|^{2_{\alpha}^{*}-2}+\left|v_{n}^{1}\right|^{2_{\alpha}^{*}-2}\right)\left|u_{0} \varphi\right| d x+\int_{\mathbb{R}^{N} \backslash B_{R}(0)}\left|u_{0}\right|^{2_{\alpha}^{*}-1}|\varphi| d x \leq C \varepsilon\|\varphi\| .
\end{aligned}
$$

On the other hand, for every $r>0$, we have

$$
\begin{aligned}
& \left|\int_{B_{R}(0)}\left(\left|u_{n}\right|^{2_{\alpha}^{*}-2} u_{n}-\left|u_{0}\right|^{2_{\alpha}^{*}-2} u-\left|v_{n}^{1}\right|^{2_{\alpha}^{*}-2} v_{n}^{1}\right) \varphi d x\right| \\
& \leq\left.\int_{B_{R}(0) \cap\left\{\left|v_{n}^{1}\right| \leq r\right\}}|| u_{n}\right|^{2_{\alpha}^{*}-2} u_{n}-\left|u_{0}\right|^{2_{\alpha}^{*}-2} u_{0}-\left|v_{n}^{1}\right|^{2_{\alpha}^{*}-2} v_{n}^{1} \mid \varphi d x \\
& \quad+\left.\int_{B_{R}(0) \cap\left\{\left|v_{n}^{1}\right| \geq r\right\}}|| u_{n}\right|^{2_{\alpha}^{*}-2} u_{n}-\left|u_{0}\right|^{2_{\alpha}^{*}-2} u_{0}-\left|v_{n}^{1}\right|^{2_{\alpha}^{*}-2} v_{n}^{1} \mid \varphi d x=: I_{1}+I_{2} .
\end{aligned}
$$

Now, there exists $r=r(R)$ such that $r\left|B_{R}(0)\right|^{1 / 2_{\alpha}^{*}} \leq \varepsilon$. Therefore, we have

$$
\begin{aligned}
I_{1} & \leq C \int_{B_{R}(0) \cap\left\{\left|v_{n}^{1}\right| \leq r\right\}}\left(\left|u_{n}\right|^{2_{\alpha}^{*}-2}+\left|u_{0}\right|^{2_{\alpha}^{*}-2}+\left|v_{n}^{1}\right|^{2_{\alpha}^{*}-2}\right)\left|v_{n}^{1} \varphi\right| d x \\
& \leq C r\left|B_{R}(0)\right|^{1 / 2_{\alpha}^{*}}\|\varphi\| \leq C \varepsilon\|\varphi\| .
\end{aligned}
$$


For such $r, R$ fixed above, $u_{n}$ converges to $u$ in measure in $B_{R}(0)$, i.e. $\left|B_{R}(0) \cap\left\{\left|v_{n}\right| \geq r\right\}\right| \rightarrow 0$ for $n \rightarrow \infty$. Therefore, for $n \geq 1$ large,

$$
\begin{aligned}
I_{2} \leq & C \int_{B_{R}(0) \cap\left\{\left|v_{n}^{1}\right| \geq r\right\}}\left(\left|u_{n}\right|^{2_{\alpha}^{*}-2}+\left|v_{n}^{1}\right|^{2_{\alpha}^{*}-2}\right)\left|u_{0} \varphi\right| d x \\
& +\int_{B_{R}(0) \cap\left\{\left|v_{n}^{1}\right| \geq r\right\}}\left|u_{0}\right|^{2_{\alpha}^{*}-1}|\varphi| d x \leq C \varepsilon\|\varphi\| .
\end{aligned}
$$

Then (5.11), (5.12) and (5.13) yield the assertion.

Lemma 5.3 (Splitting lemma III). We have

$$
\int_{\mathbb{R}^{N}} f\left(u_{n}\right) u_{n} d x=\int_{\mathbb{R}^{N}} f\left(v_{n}^{1}\right) v_{n}^{1} d x+\int_{\mathbb{R}^{N}} f\left(u_{0}\right) u_{0} d x+o_{n}(1),
$$

where $o_{n}(1) \rightarrow 0$ as $n \rightarrow \infty$. Furthermore

$$
\int_{\mathbb{R}^{N}} F\left(u_{n}\right) d x=\int_{\mathbb{R}^{N}} F\left(v_{n}^{1}\right) d x+\int_{\mathbb{R}^{N}} F\left(u_{0}\right) d x+o_{n}(1) .
$$

Proof. Since $f(t)=g(t)+t^{2_{\alpha}^{*}-1}$ for $t \geq 0$, by the standard Brezis-Lieb lemma, it suffices to prove

$$
\int_{\mathbb{R}^{N}} g\left(u_{n}\right) u_{n} d x=\int_{\mathbb{R}^{N}} g\left(v_{n}^{1}\right) v_{n}^{1} d x+\int_{\mathbb{R}^{N}} g\left(u_{0}\right) u_{0} d x+o_{n}(1),
$$

where $o_{n}(1) \rightarrow 0$ as $n \rightarrow \infty$. Fixed $\varepsilon>0$, there exists $C_{\varepsilon}>0$ such that

$$
|g(t)| \leq \varepsilon t^{2_{\alpha}^{*}-1}+C_{\varepsilon} t, \quad t \geq 0 .
$$

Then there exists $R=R(\varepsilon)>0$ large enough such that

$$
\begin{aligned}
\left|\int_{\mathbb{R}^{N}} g\left(u_{0}\right) v_{n}^{1} d x\right| & \leq \int_{B_{R}}\left|g\left(u_{0}\right) v_{n}^{1}\right| d x+\int_{B_{R}^{c}}\left|g\left(u_{0}\right) v_{n}^{1}\right| d x \\
& \leq \int_{B_{R}}\left(\varepsilon\left|u_{0}\right|^{2_{\alpha}^{*}-1}+C_{\varepsilon}\left|u_{0}\right|\right)\left|v_{n}^{1}\right| d x+\varepsilon\left(\left\|v_{n}^{1}\right\|_{2}+\left\|v_{n}^{1}\right\|_{2_{\alpha}^{*}}\right) \leq C \varepsilon+C_{\varepsilon} o_{n}(1) .
\end{aligned}
$$

and

$$
\begin{aligned}
\left|\int_{\mathbb{R}^{N}} g\left(v_{n}^{1}\right) u_{0} d x\right| & \leq \int_{B_{R}}\left|g\left(v_{n}^{1}\right) u_{0}\right| d x+\int_{B_{R}^{c}}\left|g\left(v_{n}^{1}\right) u_{0}\right| d x \\
& \leq \int_{B_{R}}\left(\varepsilon\left|v_{n}^{1}\right|^{2_{\alpha}^{*}-1}+C_{\varepsilon}\left|v_{n}^{1}\right|\right)\left|u_{0}\right| d x+\int_{B_{R}^{c}}\left(\varepsilon\left|v_{n}^{1}\right|^{2_{\alpha}^{*}-1}+C_{\varepsilon}\left|v_{n}^{1}\right|\right)\left|u_{0}\right| d x \\
& \leq C \varepsilon+C_{\varepsilon} o_{n}(1) .
\end{aligned}
$$

It follows from (5.15), (5.16) and Lemma 5.1 that

$$
\begin{aligned}
& \left|\int_{\mathbb{R}^{N}}\left(g\left(u_{n}\right) u_{n}-g\left(u_{0}\right) u_{0}-g\left(v_{n}^{1}\right) v_{n}^{1}\right) d x\right| \\
& \leq \int_{\mathbb{R}^{N}}\left|\left(g\left(u_{n}\right)-g\left(u_{0}\right)-g\left(v_{n}^{1}\right)\right) u_{n}\right| d x+\int_{\mathbb{R}^{N}}\left|g\left(v_{n}^{1}\right) u_{0}\right| d x+\int_{\mathbb{R}^{N}}\left|g\left(u_{0}\right) v_{n}^{1}\right| d x \\
& \leq o_{n}(1)\left\|u_{n}\right\|+C \varepsilon+C_{\varepsilon} o_{n}(1) .
\end{aligned}
$$

Letting $n \rightarrow \infty$ and $\varepsilon \rightarrow 0^{+}$completes the proof of the first assertion. The second assertion follows from the standard Brezis-Lieb lemma and

$$
\int_{\mathbb{R}^{N}} G\left(u_{n}\right) d x=\int_{\mathbb{R}^{N}} G\left(v_{n}^{1}\right) d x+\int_{\mathbb{R}^{N}} G\left(u_{0}\right) d x+o_{n}(1),
$$

whose proof is left to the reader. 
5.2. Profile decomposition. In the following, we give the profile decomposition of $\left\{u_{n}\right\}_{n \in \mathbb{N}}$, which plays a crucial role in getting the compactness. Since $c_{\lambda}>0$, for some $\bar{B}>0$ we have

$$
\int_{\mathbb{R}^{N}}\left|(-\Delta)^{\frac{\alpha}{2}} u_{n}\right|^{2} d x \rightarrow \bar{B}^{2}, \quad \text { as } n \rightarrow \infty .
$$

Now, for any $u \in \mathcal{H}$, let

$$
J_{\lambda}(u):=\frac{a+b \bar{B}^{2}}{2} \int_{\mathbb{R}^{N}}\left|(-\Delta)^{\frac{\alpha}{2}} u\right|^{2} d x+\frac{1}{2} \int_{\mathbb{R}^{N}} V(x)|u|^{2} d x-\lambda \int_{\mathbb{R}^{N}} F(u) d x
$$

and

$$
J_{\lambda}^{\infty}(u):=\frac{a+b \bar{B}^{2}}{2} \int_{\mathbb{R}^{N}}\left|(-\Delta)^{\frac{\alpha}{2}} u\right|^{2} d x+\frac{1}{2} \int_{\mathbb{R}^{N}} V_{\infty}|u|^{2} d x-\lambda \int_{\mathbb{R}^{N}} F(u) d x,
$$

which are respectively the corresponding functional of the following problems

$$
\left(a+b \bar{B}^{2}\right)(-\Delta)^{\alpha} u+V(x) u=f(u), \quad\left(a+b \bar{B}^{2}\right)(-\Delta)^{\alpha} u+V_{\infty} u=f(u), u \in \mathcal{H} .
$$

Here we point out that in contrast with the original problem $(\mathrm{K})$, the problems above are both non Kirchhoff. Now we take advantage of this to get the profile decomposition of $\left\{u_{n}\right\}_{n \in \mathbb{N}}$.

Lemma 5.4 (Profile decomposition). Let $\left\{u_{n}\right\}_{n \in \mathbb{N}} \subset \mathcal{H}$ be the sequence mentioned above and assume that conditions $\left(\mathrm{V}_{1}\right)-\left(\mathrm{V}_{3}\right),\left(\mathrm{f}_{1}\right)-\left(\mathrm{f}_{3}\right)$ hold and $N<4 \alpha$. Then $J_{\lambda}^{\prime}\left(u_{0}\right)=0$, and there exist a number $k \in \mathbb{N} \cup\{0\}$, nontrivial critical points $w^{1}, \ldots, w^{k}$ of $J_{\lambda}^{\infty}$ such that

(i) $\left|y_{n}^{j}\right| \rightarrow+\infty,\left|y_{n}^{j}-y_{n}^{i}\right| \rightarrow+\infty \quad$ if $i \neq j, 1 \leq i, j \leq k, n \rightarrow+\infty$,

(ii) $c_{\lambda}+\frac{b \bar{B}^{4}}{4}=J_{\lambda}\left(u_{0}\right)+\sum_{j=1}^{k} J_{\lambda}^{\infty}\left(w^{j}\right)$,

(iii) $\left\|u_{n}-u_{0}-\sum_{j=1}^{k} w^{j}\left(\cdot-y_{n}^{j}\right)\right\| \rightarrow 0$,

(iv) $\bar{B}^{2}=\left\|(-\Delta)^{\frac{\alpha}{2}} u_{0}\right\|_{2}^{2}+\sum_{j=1}^{k}\left\|(-\Delta)^{\frac{\alpha}{2}} w^{j}\right\|_{2}^{2}$.

Moreover, we agree that in the case $k=0$ the above holds without $w^{j}$. In addition, if $\left(\mathrm{V}_{4}\right)$ holds, then $k=0$ and $u_{0} \in H_{\text {rad }}^{\alpha}\left(\mathbb{R}^{N}\right)$.

Proof. Observe that, from $I_{\lambda}\left(u_{n}\right)=c_{\lambda}+o_{n}(1)$ and $I_{\lambda}^{\prime}\left(u_{n}\right) \rightarrow 0$ in $\mathcal{H}^{\prime}$, we obtain

$$
J_{\lambda}\left(u_{n}\right)=c_{\lambda}+\frac{b \bar{B}^{4}}{4}+o_{n}(1), \quad J_{\lambda}^{\prime}\left(u_{n}\right) \rightarrow 0 \quad \text { in } \mathcal{H}^{\prime}
$$

Then, it is standard to get $J_{\lambda}^{\prime}\left(u_{0}\right) \varphi=0$ for all $\varphi \in \mathcal{H}$. From Lemma 5.3, we get

$$
\begin{aligned}
\int_{\mathbb{R}^{N}} F\left(v_{n}^{1}\right) d x & =\int_{\mathbb{R}^{N}} F\left(u_{n}\right) d x-\int_{\mathbb{R}^{N}} F\left(u_{0}\right) d x+o_{n}(1), \\
\int_{\mathbb{R}^{N}} f\left(v_{n}^{1}\right) v_{n}^{1} d x & =\int_{\mathbb{R}^{N}} f\left(u_{n}\right) u_{n} d x-\int_{\mathbb{R}^{N}} f\left(u_{0}\right) u_{0} d x+o_{n}(1) .
\end{aligned}
$$

It follows that

$$
\begin{aligned}
J_{\lambda}\left(u_{n}\right) & =J_{\lambda}\left(v_{n}^{1}\right)+J_{\lambda}\left(u_{0}\right)+o_{n}(1), \\
J_{\lambda}^{\prime}\left(v_{n}^{1}\right) v_{n}^{1} & =J_{\lambda}^{\prime}\left(u_{n}\right) u_{n}-J_{\lambda}^{\prime}\left(u_{0}\right) u_{0}+o_{n}(1)=o_{n}(1) .
\end{aligned}
$$


On the other hand, by a slight variant of [10, Proposition 4.1], $u_{0}$ satisfies the Pohǒzaev identity

$$
\begin{aligned}
\frac{N-2 \alpha}{2}\left(a+b \bar{B}^{2}\right) \int_{\mathbb{R}^{N}}\left|(-\Delta)^{\frac{\alpha}{2}} u_{0}\right|^{2} d x & +\frac{1}{2} \int_{\mathbb{R}^{N}} \nabla V(x) \cdot x u_{0}^{2} d x \\
& +\frac{N}{2} \int_{\mathbb{R}^{N}} V(x) u_{0}^{2} d x-N \lambda \int_{\mathbb{R}^{N}} F\left(u_{0}\right) d x=0 .
\end{aligned}
$$

Then by $\left(\mathrm{V}_{1}\right)$ and $N<4 \alpha$, we have

$$
\begin{aligned}
N J_{\lambda}\left(u_{0}\right) & =\alpha\left(a+b \bar{B}^{2}\right) \int_{\mathbb{R}^{N}}\left|(-\Delta)^{\frac{\alpha}{2}} u_{0}\right|^{2} d x-\frac{1}{2} \int_{\mathbb{R}^{N}} \nabla V(x) \cdot x u_{0}^{2} d x \\
& \geq \alpha\left(a+b \bar{B}^{2}\right) \int_{\mathbb{R}^{N}}\left|(-\Delta)^{\frac{\alpha}{2}} u_{0}\right|^{2} d x-\frac{1}{2}\|W\|_{\frac{N}{2 \alpha}}\left\|u_{0}\right\|_{2_{\alpha}^{*}}^{2} \\
& \geq \alpha\left(a+b \bar{B}^{2}\right) \int_{\mathbb{R}^{N}}\left|(-\Delta)^{\frac{\alpha}{2}} u_{0}\right|^{2} d x-a \alpha \int_{\mathbb{R}^{N}}\left|(-\Delta)^{\frac{\alpha}{2}} u_{0}\right|^{2} d x \\
& =\alpha b \bar{B}^{2} \int_{\mathbb{R}^{N}}\left|(-\Delta)^{\frac{\alpha}{2}} u_{0}\right|^{2} d x>0,
\end{aligned}
$$

which implies that

$$
J_{\lambda}\left(u_{0}\right) \geq \frac{b \bar{B}^{2}}{4} \int_{\mathbb{R}^{N}}\left|(-\Delta)^{\frac{\alpha}{2}} u_{0}\right|^{2} d x .
$$

We claim that one of the following conclusions holds for $v_{n}^{1}$ :

(v1) $v_{n}^{1} \rightarrow 0$ in $\mathcal{H}$, or

(v2) there exist $r^{\prime}>0, \sigma>0$ and a sequence $\left\{y_{n}^{1}\right\}_{n \in \mathbb{N}} \subset \mathbb{R}^{N}$ such that

$$
\liminf _{n \rightarrow \infty} \int_{B_{r^{\prime}}\left(y_{n}^{1}\right)}\left|v_{n}^{1}\right|^{2} d x \geq \sigma>0 .
$$

Indeed, suppose that (v2) does not occur. Then for any $r>0$, we have

$$
\lim _{n \rightarrow \infty} \sup _{y \in \mathbb{R}^{N}} \int_{B_{r}(y)}\left|v_{n}^{1}\right|^{2} d x=0 .
$$

Therefore, it follows from Lemma 2.2 that $v_{n}^{1} \rightarrow 0$ in $L^{s}\left(\mathbb{R}^{N}\right)$ for $s \in\left(2,2_{\alpha}^{*}\right)$. It follows from (5.14) that for any $\varepsilon>0$, there exists $C_{\varepsilon}>0$ such that

$$
\int_{\mathbb{R}^{N}}\left|g\left(v_{n}^{1}\right) v_{n}^{1}\right| d x \leq \varepsilon\left(\int_{\mathbb{R}^{N}}\left|v_{n}^{1}\right|^{2}+\left|v_{n}^{1}\right|^{2_{\alpha}^{*}}\right) d x+C_{\varepsilon} \int_{\mathbb{R}^{N}}\left|v_{n}^{1}\right|^{q} d x .
$$

So from $v_{n}^{1} \rightarrow 0$ in $L^{q}\left(\mathbb{R}^{N}\right)$ and the arbitrariness of $\varepsilon$, we can easily obtain that

$$
\int_{\mathbb{R}^{N}} f\left(v_{n}^{1}\right) v_{n}^{1} d x=\int_{\mathbb{R}^{N}}\left(\left(v_{n}^{1}\right)^{+}\right)^{2_{\alpha}^{*}} d x+o_{n}(1) .
$$

Furthermore, from $J_{\lambda}^{\prime}\left(v_{n}^{1}\right) v_{n}^{1}=o_{n}(1)$ in (5.18), we have

$$
\left\|v_{n}^{1}\right\|^{2}+b \bar{B}^{2} \int_{\mathbb{R}^{N}}\left|(-\Delta)^{\frac{\alpha}{2}} v_{n}^{1}\right|^{2} d x=\lambda\left\|\left(v_{n}^{1}\right)^{+}\right\|_{2_{\alpha}^{*}}^{2^{*}}+o_{n}(1)
$$

In view of conditions $\left(\mathrm{V}_{2}\right)-\left(\mathrm{V}_{3}\right)$, we can check that $V_{\infty}>0$. And so we can also get

$$
\int_{\mathbb{R}^{N}} V(x)\left|v_{n}^{1}\right|^{2} d x=\int_{\mathbb{R}^{N}} V^{+}(x)\left|v_{n}^{1}\right|^{2} d x+o_{n}(1),
$$

which, together with the definition of $S_{\alpha}$ and (5.21), implies that

$$
a S_{\alpha}\left(\int_{\mathbb{R}^{N}}\left|v_{n}^{1}\right|^{2_{\alpha}^{*}} d x\right)^{\frac{2}{2_{\alpha}^{*}}}+b S_{\alpha}^{2}\left(\int_{\mathbb{R}^{N}}\left|v_{n}^{1}\right|^{2_{\alpha}^{*}} d x\right)^{\frac{4}{2_{\alpha}^{*}}} \leq \lambda \int_{\mathbb{R}^{N}}\left|v_{n}^{1}\right|^{2_{\alpha}^{*}} d x+o_{n}(1) .
$$


Let $\ell \geq 0$ be such that $\int_{\mathbb{R}^{N}}\left|v_{n}^{1}\right|^{2_{\alpha}^{*}} d x \rightarrow \ell^{N}$. If $\ell>0$, then it follows from (5.22) that

$$
K^{\prime}(\ell)=\frac{(N-2 \alpha) \ell^{-1}}{2}\left(a S_{\alpha} \ell^{N-2 \alpha}+b S_{\alpha}^{2} \ell^{2 N-4 \alpha}-\lambda \ell^{N}\right) \leq 0
$$

where $K$ has been defined in Lemma 4.1. This also implies that $\ell \geq T$ ( $T$ is the unique maximum point of $K)$. On the other hand, by (5.17) and (5.19), we have

$$
\begin{aligned}
c_{\lambda}+\frac{b \bar{B}^{4}}{4} & =\int_{\mathbb{R}^{N}}\left(\frac{a+b \bar{B}^{2}}{2}\left|(-\Delta)^{\frac{\alpha}{2}} v_{n}^{1}\right|^{2}+\frac{1}{2} V(x)\left|v_{n}^{1}\right|^{2}-\frac{\lambda}{2_{\alpha}^{*}}\left(\left(v_{n}^{1}\right)^{+}\right)^{2_{\alpha}^{*}}\right) d x+J_{\lambda}\left(u_{0}\right)+o_{n}(1) \\
& \geq \int_{\mathbb{R}^{N}}\left(\left(\frac{a}{2}+\frac{b \bar{B}^{2}}{4}\right)\left|(-\Delta)^{\frac{\alpha}{2}} v_{n}^{1}\right|^{2}+\frac{1}{2} V(x)\left|v_{n}^{1}\right|^{2}-\frac{\lambda}{2_{\alpha}^{*}}\left(\left(v_{n}^{1}\right)^{+}\right)^{2_{\alpha}^{*}}\right) d x+\frac{b \bar{B}^{4}}{4}+o_{n}(1),
\end{aligned}
$$

which, together with (5.21) and the definition of $S_{\alpha}$, implies that

$$
\begin{aligned}
c_{\lambda} & \geq\left(\frac{1}{2}-\frac{1}{2_{\alpha}^{*}}\right) a \int_{\mathbb{R}^{N}}\left|(-\Delta)^{\frac{\alpha}{2}} v_{n}^{1}\right|^{2} d x+\left(\frac{1}{4}-\frac{1}{2_{\alpha}^{*}}\right) b\left(\int_{\mathbb{R}^{N}}\left|(-\Delta)^{\frac{\alpha}{2}} v_{n}^{1}\right|^{2} d x\right)^{2}+o_{n}(1) \\
& \geq\left(\frac{1}{2}-\frac{1}{2_{\alpha}^{*}}\right) a S_{\alpha}\left(\int_{\mathbb{R}^{N}}\left|v_{n}^{1}\right|^{2_{\alpha}^{*}} d x\right)^{\frac{2}{2_{\alpha}^{*}}}+\left(\frac{1}{4}-\frac{1}{2_{\alpha}^{*}}\right) b S_{\alpha}^{2}\left(\int_{\mathbb{R}^{N}}\left|v_{n}^{1}\right|^{2_{\alpha}^{*}} d x\right)^{\frac{4}{2_{\alpha}^{*}}}+o_{n}(1) .
\end{aligned}
$$

Thus, combining $\int_{\mathbb{R}^{N}}\left|v_{n}^{1}\right|^{2_{\alpha}^{*}} d x \rightarrow \ell^{N}$ and $\ell \geq T, K^{\prime}(T)=0$, we have

$$
\begin{aligned}
c_{\lambda} & \geq\left(\frac{1}{2}-\frac{1}{2_{\alpha}^{*}}\right) a S_{\alpha} \ell^{N-2 \alpha}+\left(\frac{1}{4}-\frac{1}{2_{\alpha}^{*}}\right) b S_{\alpha}^{2} \ell^{2 N-4 \alpha} \\
& \geq\left(\frac{1}{2}-\frac{1}{2_{\alpha}^{*}}\right) a S_{\alpha} T^{N-2 \alpha}+\left(\frac{1}{4}-\frac{1}{2_{\alpha}^{*}}\right) b S_{\alpha}^{2} T^{2 N-4 \alpha} \\
& =\frac{1}{2} a S_{\alpha} T^{N-2 \alpha}+\frac{1}{4} b S_{\alpha}^{2} T^{2 N-4 \alpha}-\frac{\lambda}{2_{\alpha}^{*}} T^{N}=c_{\lambda}^{*},
\end{aligned}
$$

contradicting $c_{\lambda}<c_{\lambda}^{*}$. Hence, $\ell=0$. It follows from (5.21) that $\left\|v_{n}^{1}\right\| \rightarrow 0$, that is, $u_{n} \rightarrow u_{0}$ in $\mathcal{H}$. Then Lemma 5.4 hold with $k=0$ if (v2) does not occur. In particular, if we assume $\left(\mathrm{V}_{4}\right)$ holds, then by Corollary 3.5, $\left\|u_{n}-\left|u_{n}\right|^{*}\right\|_{2_{\alpha}^{*}} \rightarrow 0$. Obviously, $\left\{\left|u_{n}\right|^{*}\right\}_{n \in \mathbb{N}} \subset H_{\text {rad }}^{\alpha}\left(\mathbb{R}^{N}\right)$ is bounded and $\left\|u_{n}-\left|u_{n}\right|^{*}\right\|_{q} \rightarrow 0$ for $q \in\left(2,2_{\alpha}^{*}\right)$. Since $\left\{\left|u_{n}\right|^{*}\right\}_{n \in \mathbb{N}}$ has a strongly convergent subsequence in $L^{q}\left(\mathbb{R}^{N}\right)$ for $q \in\left(2,2_{\alpha}^{*}\right)$, without loss of generality, we assume that $u_{n} \rightarrow u_{0}$ in $L^{q}\left(\mathbb{R}^{N}\right)$ for $q \in\left(2,2_{\alpha}^{*}\right)$ and $u_{0}=u_{0}^{*}$. As a consequence, (v2) does not hold and as above, $u_{n} \rightarrow u_{0}$ in $\mathcal{H}$.

In the following, otherwise, suppose that (v2) holds, that is (5.20) holds. Consider $v_{n}^{1}\left(\cdot+y_{n}^{1}\right)$. The boundedness of $\left\{v_{n}^{1}\right\}_{n \in \mathbb{N}}$ and (5.20) imply that $v_{n}^{1}\left(\cdot+y_{n}^{1}\right) \rightarrow w^{1} \neq 0$ in $\mathcal{H}$. Thus, it follows from $v_{n}^{1} \rightarrow 0$ in $\mathcal{H}$ that $\left\{y_{n}^{1}\right\}_{n \in \mathbb{N}}$ is unbounded and, up to a subsequence, $\left|y_{n}^{1}\right| \rightarrow+\infty$. Let us prove that $\left(J_{\lambda}^{\infty}\right)^{\prime}\left(w^{1}\right)=0$. It suffices to show that $\left(J_{\lambda}^{\infty}\right)^{\prime}\left(v_{n}^{1}\left(\cdot+y_{n}^{1}\right)\right) \varphi \rightarrow 0$ for any $\varphi \in C_{0}^{\infty}\left(\mathbb{R}^{N}\right)$. Combining Lemma 5.1 and Lemma 5.2, we obtain

$$
\left|J_{\lambda}^{\prime}\left(u_{n}\right) \varphi-J_{\lambda}^{\prime}\left(u_{0}\right) \varphi-J_{\lambda}^{\prime}\left(v_{n}^{1}\right) \varphi\right| \leq o_{n}(1)\|\varphi\|, \quad \forall \varphi \in C_{0}^{\infty}\left(\mathbb{R}^{N}\right),
$$

which implies that $\left|J_{\lambda}^{\prime}\left(v_{n}^{1}\right) \varphi\right| \leq o_{n}(1)\|\varphi\|$, for all $\varphi \in C_{0}^{\infty}\left(\mathbb{R}^{N}\right)$, as $n \rightarrow \infty$. Notice that

$$
\begin{aligned}
J_{\lambda}^{\prime}\left(v_{n}^{1}\right) \varphi\left(\cdot-y_{n}^{1}\right)= & \frac{C(n, \alpha)}{2}\left(a+b \bar{B}^{2}\right) \int_{\mathbb{R}^{2 N}} \frac{\left(v_{n}^{1}(x)-v_{n}^{1}(y)\right)\left(\varphi\left(x-y_{n}^{1}\right)-\varphi\left(y-y_{n}^{1}\right)\right)}{|x-y|^{N+2 \alpha}} d x d y \\
& +\int_{\mathbb{R}^{N}} V(x) v_{n}^{1}(x) \varphi\left(x-y_{n}^{1}\right) d x-\lambda \int_{\mathbb{R}^{N}} g\left(v_{n}^{1}(x)\right) \varphi\left(x-y_{n}^{1}\right) d x \\
& -\lambda \int_{\mathbb{R}^{N}}\left(\left(v_{n}^{1}(x)\right)^{+}\right)^{2_{\alpha}^{*}-1} \varphi\left(x-y_{n}^{1}\right) d x=o_{n}(1)\left\|\varphi\left(\cdot-y_{n}^{1}\right)\right\|=o_{n}(1)\|\varphi\| .
\end{aligned}
$$


Thus, as $n \rightarrow \infty$, it follows that

$$
\begin{aligned}
& \frac{C(n, \alpha)}{2}\left(a+b \bar{B}^{2}\right) \int_{\mathbb{R}^{2 N}} \frac{\left(v_{n}^{1}\left(x+y_{n}^{1}\right)-v_{n}\left(y+y_{n}^{1}\right)\right)(\varphi(x)-\varphi(y))}{|x-y|^{N+2 \alpha}} d x d y+ \\
& \int_{\mathbb{R}^{N}} V\left(x+y_{n}^{1}\right) v_{n}^{1}\left(x+y_{n}^{1}\right) \varphi(x) d x-\lambda \int_{\mathbb{R}^{N}} g\left(v_{n}^{1}\left(x+y_{n}^{1}\right)\right) \varphi(x) d x \\
& -\int_{\mathbb{R}^{N}}\left(\left(v_{n}^{1}\left(x+y_{n}^{1}\right)\right)^{+}\right)^{2_{\alpha}^{*}-1} \varphi(x) d x=o_{n}(1)\|\varphi\| .
\end{aligned}
$$

Since $\left|y_{n}^{1}\right| \rightarrow \infty$ and $\varphi \in C_{0}^{\infty}\left(\mathbb{R}^{N}\right)$, we obtain

$$
\int_{\mathbb{R}^{N}}\left(V\left(x+y_{n}^{1}\right)-V_{\infty}\right) v_{n}^{1}\left(x+y_{n}^{1}\right) \varphi(x) d x \rightarrow 0 .
$$

Thus, combining (5.23) and (5.24), we have for any $\varphi \in C_{0}^{\infty}\left(\mathbb{R}^{N}\right)$,

$$
\begin{aligned}
\left(J_{\lambda}^{\infty}\right)^{\prime}\left(v_{n}^{1}\left(\cdot+y_{n}^{1}\right)\right) \varphi= & \frac{C(n, \alpha)}{2}\left(a+b \bar{B}^{2}\right) \int_{\mathbb{R}^{2 N}} \frac{\left(v_{n}^{1}\left(x+y_{n}^{1}\right)-v_{n}\left(y+y_{n}^{1}\right)\right)(\varphi(x)-\varphi(y))}{|x-y|^{N+2 \alpha}} d x d y \\
& +\int_{\mathbb{R}^{N}} V_{\infty} v_{n}^{1}\left(x+y_{n}^{1}\right) \varphi(x) d x-\lambda \int_{\mathbb{R}^{N}} g\left(v_{n}^{1}\left(x+y_{n}^{1}\right)\right) \varphi(x) d x \\
& -\lambda \int_{\mathbb{R}^{N}}\left(\left(v_{n}^{1}\left(x+y_{n}^{1}\right)\right)^{+}\right)^{2_{\alpha}^{*}-1} \varphi(x) d x=o_{n}(1) .
\end{aligned}
$$

Then, $\left(J_{\lambda}^{\infty}\right)^{\prime}\left(w^{1}\right)=0$. Finally, let us set

$$
v_{n}^{2}(x)=v_{n}^{1}(x)-w^{1}\left(x-y_{n}^{1}\right),
$$

then $v_{n}^{2} \rightarrow 0$ in $\mathcal{H}$. Since $V(x) \rightarrow V_{\infty}$ as $|x| \rightarrow \infty$ and $v_{n}^{1} \rightarrow 0$ strongly in $L_{\text {loc }}^{2}\left(\mathbb{R}^{N}\right)$, we have

$$
\int_{\mathbb{R}^{N}}\left(V(x)-V_{\infty}\right)\left(v_{n}^{1}\right)^{2} d x=o_{n}(1) .
$$

It follows that

$$
\begin{aligned}
& \int_{\mathbb{R}^{N}} V(x)\left|v_{n}^{2}\right|^{2} d x=\int_{\mathbb{R}^{N}} V(x)\left|v_{n}^{1}\right|^{2} d x+\int_{\mathbb{R}^{N}} V\left(x+y_{n}^{1}\right)\left|w^{1}(x)\right|^{2} d x \\
&-2 \int_{\mathbb{R}^{N}} V\left(x+y_{n}^{1}\right) v_{n}^{1}\left(x+y_{n}^{1}\right) w^{1}(x) d x \\
&=\int_{\mathbb{R}^{N}} V_{\infty}\left|u_{n}\right|^{2} d x-\int_{\mathbb{R}^{N}} V_{\infty}\left|u_{0}\right|^{2} d x-\int_{\mathbb{R}^{N}} V_{\infty}\left|w^{1}\right|^{2} d x+o_{n}(1) \\
&=\int_{\mathbb{R}^{N}} V(x)\left|u_{n}\right|^{2} d x-\int_{\mathbb{R}^{N}} V(x)\left|u_{0}\right|^{2} d x-\int_{\mathbb{R}^{N}} V_{\infty}\left|w^{1}\right|^{2} d x+o_{n}(1), \\
&\left\{\begin{array}{c}
\left\|v_{n}^{2}\right\|^{2}=\left\|u_{n}\right\|^{2}-\left\|u_{0}\right\|^{2}-\left\|w^{1}\left(\cdot-y_{n}^{1}\right)\right\|^{2}+o_{n}(1), \\
\left\|v_{n}^{2}\right\|_{2_{\alpha}^{*}}^{2^{*}}=\left\|u_{n}\right\|_{2_{\alpha}^{*}}^{2_{\alpha}^{*}}-\left\|u_{0}\right\|_{2_{\alpha}^{*}}^{2_{\alpha}^{*}}-\left\|w^{1}\right\|_{2_{\alpha}^{2}}^{2_{\alpha}^{*}}+o_{n}(1), \\
\int_{\mathbb{R}^{N}} G\left(v_{n}^{2}\right) d x=\int_{\mathbb{R}^{N}} G\left(u_{n}\right) d x-\int_{\mathbb{R}^{N}} G\left(u_{0}\right) d x-\int_{\mathbb{R}^{N}} G\left(w^{1}\right) d x .
\end{array}\right.
\end{aligned}
$$

Similar to (5.1), we also have

$$
\int_{\mathbb{R}^{N}} g\left(v_{n}^{2}\right) \varphi d x=\int_{\mathbb{R}^{N}} g\left(u_{n}\right) \varphi d x-\int_{\mathbb{R}^{N}} g\left(u_{0}\right) \varphi d x-\int_{\mathbb{R}^{N}} g\left(w^{1}\left(\cdot-y_{n}^{1}\right)\right) \varphi d x+o_{n}(1)\|\varphi\|,
$$


for any $\varphi \in C_{0}^{\infty}\left(\mathbb{R}^{N}\right)$. Combining (5.26), (5.27), (5.28) and (5.29), we deduce that

(1) $J_{\lambda}\left(v_{n}^{2}\right)=J_{\lambda}\left(u_{n}\right)-J_{\lambda}\left(u_{0}\right)-J_{\lambda}^{\infty}\left(w^{1}\right)+o_{n}(1)$,

(2) $J_{\lambda}^{\prime}\left(v_{n}^{2}\right) \varphi=J_{\lambda}^{\prime}\left(u_{n}\right) \varphi-J_{\lambda}^{\prime}\left(u_{0}\right) \varphi-\left(J_{\lambda}^{\infty}\right)^{\prime}\left(w^{1}\left(\cdot-y_{n}^{1}\right)\right) \varphi+o_{n}(1)\|\varphi\|=o_{n}(1)\|\varphi\|$,

(3) $J_{\lambda}^{\infty}\left(v_{n}^{2}\right)=J_{\lambda}^{\infty}\left(v_{n}^{1}\right)-J_{\lambda}^{\infty}\left(w^{1}\right)+o_{n}(1)$

for any $\varphi \in C_{0}^{\infty}\left(\mathbb{R}^{N}\right)$. Thus, we get

$$
J_{\lambda}\left(v_{n}^{2}\right)=c_{\lambda}+\frac{b \bar{B}^{4}}{4}-J_{\lambda}\left(u_{0}\right)-J_{\lambda}^{\infty}\left(w^{1}\right)+o_{n}(1)<c_{\lambda}^{*}+\frac{b \bar{B}^{4}}{4} .
$$

Remark that one of (v1) and (v2) holds for $v_{n}^{2}$. If $v_{n}^{2} \rightarrow 0$ in $\mathcal{H}$, then Lemma 5.4 holds with $k=1$. Otherwise, $\left\{v_{n}^{2}\right\}$ is non-vanishing, that is, (v2) holds for $v_{n}^{2}$. Similarly, we repeat the arguments. By iterating this procedure we obtain sequences of points $\left\{y_{n}^{j}\right\} \subset \mathbb{R}^{N}$ such that $\left|y_{n}^{j}\right| \rightarrow+\infty$, $\left|y_{n}^{j}-y_{n}^{i}\right| \rightarrow+\infty$ if $i \neq j$ as $n \rightarrow+\infty$ and $v_{n}^{j}=v_{n}^{j-1}-w^{j-1}\left(x-y_{n}^{j-1}\right)$ (like (5.25)) with $j \geq 2$ such that $v_{n}^{j} \rightarrow 0$ in $\mathcal{H},\left(J_{\lambda}^{\infty}\right)^{\prime}\left(w^{j}\right)=0$. Using the properties of the weak convergence, we have

$$
\begin{aligned}
& \text { (a) }\left\|u_{n}\right\|^{2}-\left\|u_{0}\right\|^{2}-\sum_{j=1}^{k}\left\|w^{j}\left(\cdot-y_{n}^{j}\right)\right\|^{2}=\left\|u_{n}-u_{0}-\sum_{j=1}^{k} w^{j}\left(\cdot-y_{n}^{j}\right)\right\|^{2}+o(1), \\
& \text { (b) } J_{\lambda}\left(u_{n}\right) \rightarrow J_{\lambda}\left(u_{0}\right)+\sum_{j=1}^{k} J_{\lambda}^{\infty}\left(w^{j}\right)+J_{\lambda}^{\infty}\left(v_{n}^{k+1}\right) .
\end{aligned}
$$

Note that there is $\rho>0$ such that $\|w\| \geq \rho$ for every nontrivial critical point $w$ of $J_{\lambda}^{\infty}$ and $\left\{u_{n}\right\}_{n \in \mathbb{N}}$ is bounded in $\mathcal{H}$. By (5.30)(a), the iteration stops at some $k$. That is, $v_{n}^{k+1} \rightarrow 0$ in $\mathcal{H}$. The proof is complete.

\section{Proof of the MAIN RESUlts}

In order to obtain the existence of ground state solutions of problem (K), our strategy is that we firstly obtain the existence nontrivial solutions of the perturbed problem, then as $\lambda$ goes to 1 , we get a nontrivial solution of the original problem. Finally, thanks to the profile decomposition of the (PS)-sequence, we obtain the existence of ground state solutions of problem (K).

\subsection{Nontrivial critical points of $I_{\lambda}$.}

Lemma 6.1. Assume that $\left(\mathrm{V}_{1}\right)-\left(\mathrm{V}_{3}\right)$ and $\left(\mathrm{f}_{1}\right)-\left(\mathrm{f}_{3}\right)$ hold. For almost every $\lambda \in[1 / 2,1]$, there exists $u_{\lambda} \in \mathcal{H} \backslash\{0\}$ such that $I_{\lambda}\left(u_{\lambda}\right)=c_{\lambda}$ and $I_{\lambda}^{\prime}\left(u_{\lambda}\right)=0$. In addition, if $\left(\mathrm{V}_{4}\right)$ holds, then $u_{\lambda} \in H_{\mathrm{rad}}^{\alpha}\left(\mathbb{R}^{N}\right)$.

Proof. For almost all $\lambda \in[1 / 2,1]$, there is a bounded sequence $\left\{u_{n}\right\}_{n \in \mathbb{N}} \subset \mathcal{H}$ such that $I_{\lambda}\left(u_{n}\right) \rightarrow c_{\lambda}$, $I_{\lambda}^{\prime}\left(u_{n}\right) \rightarrow 0$. From Lemma 5.4, up to a subsequence, there exist $u_{0} \in \mathcal{H}$ and $\bar{B}>0$ such that

$$
u_{n} \rightarrow u_{0} \quad \text { in } \mathcal{H}, \quad \int_{\mathbb{R}^{N}}\left|(-\Delta)^{\frac{\alpha}{2}} u_{n}\right|^{2} d x \rightarrow \bar{B}^{2}, \text { as } n \rightarrow \infty
$$

and $J_{\lambda}^{\prime}\left(u_{0}\right)=0$. Furthermore, there exist $k \in \mathbb{N} \cup\{0\}$, nontrivial critical points $w^{1}, \ldots, w^{k}$ of $J_{\lambda}^{\infty}$ and $k$ sequences of points $\left\{y_{n}^{j}\right\} \subset \mathbb{R}^{N}, 1 \leq j \leq k$, such that

$$
\left\|u_{n}-u_{0}-\sum_{j=1}^{k} w^{j}\left(\cdot-y_{n}^{j}\right)\right\| \rightarrow 0, \quad c_{\lambda}+\frac{b \bar{B}^{4}}{4}=J_{\lambda}\left(u_{0}\right)+\sum_{j=1}^{k} J_{\lambda}^{\infty}\left(w^{j}\right)
$$


and

$$
\bar{B}^{2}=\left\|(-\Delta)^{\frac{\alpha}{2}} u_{0}\right\|_{2}^{2}+\sum_{j=1}^{k}\left\|(-\Delta)^{\frac{\alpha}{2}} w^{j}\right\|_{2}^{2} .
$$

Now we claim that if $u_{0} \neq 0$, then by $N<4 \alpha$,

$$
J_{\lambda}\left(u_{0}\right)>\frac{b \bar{B}^{2}}{4} \int_{\mathbb{R}^{N}}\left|(-\Delta)^{\frac{\alpha}{2}} u_{0}\right|^{2} d x .
$$

Indeed, since $J_{\lambda}^{\prime}\left(u_{0}\right)=0$, similar as in [10], we get

$$
\begin{aligned}
\bar{P}_{\lambda}\left(u_{0}\right):= & \frac{N-2 \alpha}{2}\left(a+b \bar{B}^{2}\right) \int_{\mathbb{R}^{N}}\left|(-\Delta)^{\frac{\alpha}{2}} u_{0}\right|^{2} d x+\frac{N}{2} \int_{\mathbb{R}^{N}} V(x) u_{0}^{2} d x \\
& +\frac{1}{2} \int_{\mathbb{R}^{N}}(\nabla V(x), x) u_{0}^{2} d x-N \lambda \int_{\mathbb{R}^{N}} F\left(u_{0}\right) d x=0 .
\end{aligned}
$$

By hypothesis $\left(\mathrm{V}_{1}\right)$ we have

$$
J_{\lambda}\left(u_{0}\right)=\frac{\alpha}{N}\left(a+b \bar{B}^{2}\right) \int_{\mathbb{R}^{N}}\left|(-\Delta)^{\frac{\alpha}{2}} u_{0}\right|^{2} d x-\frac{1}{2 N} \int_{\mathbb{R}^{N}} \nabla V(x) \cdot x u_{0}^{2} d x>\frac{\alpha}{N} b \bar{B}^{2} \int_{\mathbb{R}^{N}}\left|(-\Delta)^{\frac{\alpha}{2}} u_{0}\right|^{2} d x,
$$

which implies that $(6.3)$ holds. For each nontrivial critical point $w^{j},(j=1, \ldots, k)$ of $J_{\lambda}^{\infty}$,

$$
\frac{N-2 \alpha}{2}\left(a+b \bar{B}^{2}\right) \int_{\mathbb{R}^{N}}\left|(-\Delta)^{\frac{\alpha}{2}} w^{j}\right|^{2} d x+\frac{N}{2} \int_{\mathbb{R}^{N}} V_{\infty}\left|w^{j}\right|^{2} d x-N \lambda \int_{\mathbb{R}^{N}} F\left(w^{j}\right) d x=0 .
$$

Then it follows from (6.2) that

$$
\begin{aligned}
& \frac{a(N-2 \alpha)}{2} \int_{\mathbb{R}^{N}}\left|(-\Delta)^{\frac{\alpha}{2}} w^{j}\right|^{2} d x+\frac{b(N-2 \alpha)}{2}\left(\int_{\mathbb{R}^{N}}\left|(-\Delta)^{\frac{\alpha}{2}} w^{j}\right|^{2} d x\right)^{2} \\
& +\frac{N}{2} \int_{\mathbb{R}^{N}} V_{\infty}\left|w^{j}\right|^{2} d x-N \lambda \int_{\mathbb{R}^{N}} F\left(w^{j}\right) d x \leq 0 .
\end{aligned}
$$

Then there exists $t_{j} \in(0,1]$ such that

$$
\begin{aligned}
& \frac{a t_{j}^{N-2 \alpha}}{2}(N-2 \alpha) \int_{\mathbb{R}^{N}}\left|(-\Delta)^{\frac{\alpha}{2}} w^{j}\right|^{2} d x+\frac{b t_{j}^{2 N-4 \alpha}}{2}(N-2 \alpha)\left(\int_{\mathbb{R}^{N}}\left|(-\Delta)^{\frac{\alpha}{2}} w^{j}\right|^{2} d x\right)^{2} \\
& +\frac{N t_{j}^{N}}{2} \int_{\mathbb{R}^{N}} V_{\infty}\left|w^{j}\right|^{2} d x-N t_{j}^{N} \lambda \int_{\mathbb{R}^{N}} F\left(w^{j}\right) d x=0 .
\end{aligned}
$$

That is, $w^{j}\left(\cdot / t_{j}\right)$ satisfies the identity $P_{\lambda}(u)=0$ and it follows from Lemma 4.3 that there exists $\gamma_{\lambda} \in C([0,1], \mathcal{H})$ such that $\gamma_{\lambda}(0)=0, I_{\lambda}^{\infty}\left(\gamma_{\lambda}(1)\right)<0, w^{j} \in \gamma_{\lambda}([0,1])$ and

$$
I_{\lambda}^{\infty}\left(w^{j}\left(\cdot / t_{j}\right)\right)=\max _{t \in[0,1]} I_{\lambda}^{\infty}\left(\gamma_{\lambda}(t)\right) .
$$

By hypothesis $\left(\mathrm{V}_{2}\right)$, we have $\max _{t \in[0,1]} I_{\lambda}^{\infty}\left(\gamma_{\lambda}(t)\right) \geq \max _{t \in[0,1]} I_{\lambda}\left(\gamma_{\lambda}(t)\right)$, which, by the definition of $c_{\lambda}$, implies that $I_{\lambda}^{\infty}\left(w^{j}\left(\dot{\overline{t_{j}}}\right)\right) \geq c_{\lambda}$. In particular, if $V(x) \not \equiv V_{\infty}$, then

$$
I_{\lambda}^{\infty}\left(w^{j}\left(\cdot / t_{j}\right)\right)>c_{\lambda}
$$


So by (6.4) we have

$$
\begin{aligned}
J_{\lambda}^{\infty}\left(w^{j}\right)= & J_{\lambda}^{\infty}\left(w^{j}\right)-\frac{1}{N} P_{\lambda}\left(w^{j}\right)=\left(a+b \bar{B}^{2}\right)\left(\frac{1}{2}-\frac{1}{2_{\alpha}^{*}}\right) \int_{\mathbb{R}^{N}}\left|(-\Delta)^{\frac{\alpha}{2}} w^{j}\right|^{2} d x \\
\geq & \left(\frac{1}{2}-\frac{1}{2_{\alpha}^{*}}\right) a \int_{\mathbb{R}^{N}}\left|(-\Delta)^{\frac{\alpha}{2}} w^{j}\left(\frac{x}{t_{j}}\right)\right|^{2} d x \\
& +\left(\frac{1}{4}-\frac{1}{2_{\alpha}^{*}}\right) b\left(\int_{\mathbb{R}^{N}}\left|(-\Delta)^{\frac{\alpha}{2}} w^{j}\left(\frac{x}{t_{j}}\right)\right|^{2} d x\right)^{2}+\frac{b \bar{B}^{2}}{4} \int_{\mathbb{R}^{N}}\left|(-\Delta)^{\frac{\alpha}{2}} w^{j}\right|^{2} d x \\
= & I_{\lambda}^{\infty}\left(w^{j}\left(\frac{\dot{\sigma}}{t_{j}}\right)\right)-\frac{1}{N} P_{\lambda}\left(w^{j}\left(\frac{\dot{t}}{t_{j}}\right)\right)+\frac{b \bar{B}^{2}}{4} \int_{\mathbb{R}^{N}}\left|(-\Delta)^{\frac{\alpha}{2}} w^{j}\right|^{2} d x \\
= & I_{\lambda}^{\infty}\left(w^{j}\left(\frac{\dot{\cdot}}{t_{j}}\right)\right)+\frac{b \bar{B}^{2}}{4} \int_{\mathbb{R}^{N}}\left|(-\Delta)^{\frac{\alpha}{2}} w^{j}\right|^{2} d x
\end{aligned}
$$

and then we conclude that

$$
J_{\lambda}^{\infty}\left(w^{j}\right) \geq c_{\lambda}+\frac{b \bar{B}^{2}}{4} \int_{\mathbb{R}^{N}}\left|(-\Delta)^{\frac{\alpha}{2}} w^{j}\right|^{2} d x
$$

where the inequality is strict if $V(x) \not \equiv V_{\infty}$. Then by formulas (6.2)-(6.3),

$$
c_{\lambda}+\frac{b \bar{B}^{4}}{4}=J_{\lambda}\left(u_{0}\right)+\sum_{j=1}^{k} J_{\lambda}^{\infty}\left(w^{j}\right) \geq k c_{\lambda}+\frac{b \bar{B}^{4}}{4},
$$

where the inequality is strict if $V(x) \not \equiv V_{\infty}$. It follows that either $k=0$ or $k=1$. If $k=0$, we are done. In particular, if $\left(\mathrm{V}_{4}\right)$ holds, then $k=0$ and $u_{0} \in H_{\mathrm{rad}}^{\alpha}\left(\mathbb{R}^{N}\right)$. Then $I_{\lambda}\left(u_{0}\right)=J_{\lambda}\left(u_{0}\right)-\frac{b \bar{B}^{4}}{4}=c_{\lambda}$ and $I_{\lambda}^{\prime}\left(u_{0}\right)=J_{\lambda}^{\prime}\left(u_{0}\right)=0$. We are done. If $k=1$ and $u_{0} \neq 0$, then it follows from (6.3) and (6.7)

$$
c_{\lambda}+\frac{b \bar{B}^{4}}{4}=J_{\lambda}\left(u_{0}\right)+\sum_{j=1}^{k} J_{\lambda}^{\infty}\left(w^{j}\right)>c_{\lambda}+\frac{b \bar{B}^{4}}{4},
$$

which is a contradiction. So $u_{0}=0, k=1$ and $\bar{B}^{2}=\left\|(-\Delta)^{\frac{\alpha}{2}} w^{1}\right\|_{2}^{2}$. It follows from (6.1) and (6.2) that

$$
\left\|u_{n}-w^{1}\left(\cdot-y_{n}^{1}\right)\right\| \rightarrow 0, \quad c_{\lambda}+\frac{b \bar{B}^{4}}{4}=J_{\lambda}^{\infty}\left(w^{1}\right)
$$

Since if $V(x) \not \equiv V_{\infty}$, then by (6.5)-(6.6),

$$
J_{\lambda}^{\infty}\left(w^{1}\right) \geq I_{\lambda}^{\infty}\left(w^{1}\left(\frac{\cdot}{t_{1}}\right)\right)+\frac{b \bar{B}^{4}}{4}>c_{\lambda}+\frac{b \bar{B}^{4}}{4},
$$

which is a contradiction. Then $V(x) \equiv V_{\infty}$ and $u_{n} \rightarrow w^{1}$ strongly in $\mathcal{H}$. Therefore, $w^{1}$ is a nontrivial critical point of $I_{\lambda}^{\infty}$ and $I^{\infty}\left(u_{0}\right)=c_{\lambda}$. The proof is completed.

6.2. Completion of the proof. Choosing a sequence $\left\{\lambda_{n}\right\}_{n \in \mathbb{N}} \subset\left[\frac{1}{2}, 1\right]$ satisfying $\lambda_{n} \rightarrow 1$, we find a sequence of nontrivial critical points $\left\{u_{\lambda_{n}}\right\}_{n \in \mathbb{N}}$ (still denoted by $\left\{u_{n}\right\}_{n \in \mathbb{N}}$ ) of $I_{\lambda_{n}}$ and $I_{\lambda_{n}}\left(u_{n}\right)=c_{\lambda_{n}}$. In particular, if $\left(\mathrm{V}_{4}\right)$ holds, then $\left\{u_{n}\right\}_{n \in \mathbb{N}} \subset H_{\text {rad }}^{\alpha}\left(\mathbb{R}^{N}\right)$. Now we show that $\left\{u_{n}\right\}$ is bounded in $\mathcal{H}$. Remark that $u_{n}$ satisfies the Pohožaev identity as follows

$$
\begin{aligned}
& \frac{N-2 \alpha}{2} \int_{\mathbb{R}^{N}} a\left|(-\Delta)^{\frac{\alpha}{2}} u_{n}\right|^{2} d x+\frac{N-2 \alpha}{2} b\left(\int_{\mathbb{R}^{N}}\left|(-\Delta)^{\frac{\alpha}{2}} u_{n}\right|^{2} d x\right)^{2} \\
& +\frac{N}{2} \int_{\mathbb{R}^{N}} V(x) u_{n}^{2} d x+\frac{1}{2} \int_{\mathbb{R}^{N}} \nabla V(x) \cdot x u_{n}^{2} d x-N \lambda \int_{\mathbb{R}^{N}} F\left(u_{n}\right) d x=0 .
\end{aligned}
$$


It follows that

$$
N I_{\lambda_{n}}\left(u_{n}\right)=\alpha \int_{\mathbb{R}^{N}} a\left|(-\Delta)^{\frac{\alpha}{2}} u_{n}\right|^{2} d x+\left(\alpha-\frac{N}{4}\right) b\left(\int_{\mathbb{R}^{N}}\left|(-\Delta)^{\frac{\alpha}{2}} u_{n}\right|^{2} d x\right)^{2}-\frac{1}{2} \int_{\mathbb{R}^{N}} \nabla V(x) \cdot x u_{n}^{2} d x .
$$

Since $c_{\lambda}^{*}$ is continuous on $\lambda, I_{\lambda_{n}}\left(u_{n}\right)=c_{\lambda_{n}}+o_{n}(1)<c_{\lambda_{n}}^{*}$. It follows from $\left(\mathrm{V}_{1}\right)$ that there is a positive number $\kappa \in(0,2 a \alpha)$ such that $\|W\|_{\frac{N}{2 \alpha}} \leq \kappa S_{\alpha}$. Hence,

$$
\left(a \alpha-\frac{\kappa}{2}\right) \int_{\mathbb{R}^{N}}\left|(-\Delta)^{\frac{\alpha}{2}} u_{n}\right|^{2} d x \leq N I_{\lambda_{n}}\left(u_{n}\right),
$$

which implies that $\int_{\mathbb{R}^{N}} a\left|(-\Delta)^{\frac{\alpha}{2}} u_{n}\right|^{2} d x$ is bounded from above. By $\left(\mathrm{V}_{3}\right),\left(\mathrm{f}_{1}\right)-\left(\mathrm{f}_{2}\right)$ and $I_{\lambda_{n}}^{\prime}\left(u_{n}\right) u_{n}=$ 0 , there is $\nu>0$ such that for any $\varepsilon>0$, there exists $C_{\varepsilon}>0$ with

$$
\nu \int_{\mathbb{R}^{N}} u_{n}^{2} d x \leq \int_{\mathbb{R}^{N}} a\left|(-\Delta)^{\frac{\alpha}{2}} u_{n}\right|^{2} d x+\int_{\mathbb{R}^{N}} V(x) u_{n}^{2} d x \leq \varepsilon \int_{\mathbb{R}^{N}} u_{n}^{2} d x+C_{\varepsilon} \int_{\mathbb{R}^{N}} u_{n}^{2_{\alpha}^{*}} d x,
$$

which yields that $\left\{u_{n}\right\}_{n \in \mathbb{N}}$ is bounded in $L^{2}\left(\mathbb{R}^{N}\right)$. Then $\left\{u_{n}\right\}_{n \in \mathbb{N}}$ is bounded in $\mathcal{H}$. By Theorem 3.1 ,

$$
\lim _{n \rightarrow \infty} I\left(u_{n}\right)=\lim _{n \rightarrow \infty}\left(I_{\lambda_{n}}\left(u_{n}\right)+\left(\lambda_{n}-1\right) \int_{\mathbb{R}^{N}} F\left(u_{n}\right) d x\right)=\lim _{n \rightarrow \infty} c_{\lambda_{n}}=c_{1}
$$

and for any $\varphi \in C_{0}^{\infty}\left(\mathbb{R}^{N}\right)$,

$$
\lim _{n \rightarrow \infty} I^{\prime}\left(u_{n}\right) \varphi=\lim _{n \rightarrow \infty}\left(I_{\lambda_{n}}^{\prime}\left(u_{n}\right) \varphi+\left(\lambda_{n}-1\right) \int_{\mathbb{R}^{N}} f\left(u_{n}\right) \varphi d x\right)=0 .
$$

That is, $\left\{u_{n}\right\}_{n \in \mathbb{N}}$ is a bounded Palais-Smale sequence for $I$ at level $c_{1}$. Then by Lemma 6.1, there is a nontrivial critical point $u_{0} \in \mathcal{H}$ (radial, if $\left(\mathrm{V}_{4}\right)$ holds) for $I$ and $I\left(u_{0}\right)=c_{1}$. Set

$$
\nu=\inf \left\{I(u): u \in \mathcal{H} \backslash\{0\}, I^{\prime}(u)=0\right\} .
$$

Of course $0<\nu \leq I\left(u_{0}\right)=c_{1}<\infty$. By the definition of $\nu$, there is $\left\{u_{n}\right\}_{n \in \mathbb{N}} \subset \mathcal{H}$ with $I\left(u_{n}\right) \rightarrow \nu$ and $I^{\prime}\left(u_{n}\right)=0$. We deduce that $\left\{u_{n}\right\}_{n \in \mathbb{N}}$ is bounded in $\mathcal{H}$. Up to a sequence, for some $\bar{B}>0$,

$$
\int_{\mathbb{R}^{N}}\left|(-\Delta)^{\frac{\alpha}{2}} u_{n}\right|^{2} d x \rightarrow \bar{B}^{2}
$$

Let us set $J(u):=J_{1}(u)$ and $J^{\infty}(u):=J_{1}^{\infty}(u)$, for any $u \in \mathcal{H}$. From Lemma 5.4 there exists $u_{0} \in \mathcal{H}$ such that $u_{n} \rightarrow u_{0}$ in $\mathcal{H}$ and $J^{\prime}\left(u_{0}\right)=0$. Furthermore, there exist $k \in \mathbb{N} \cup\{0\}$, nontrivial critical points $w^{1}, \ldots, w^{k}$ of $J^{\infty}$ and $k$ sequences of points $\left\{y_{n}^{j}\right\}_{n \in \mathbb{N}} \subset \mathbb{R}^{N}, 1 \leq j \leq k$, such that

$$
\left\|u_{n}-u_{0}-\sum_{j=1}^{k} w^{j}\left(\cdot-y_{n}^{j}\right)\right\| \rightarrow 0, \quad \nu+\frac{b \bar{B}^{4}}{4}=J\left(u_{0}\right)+\sum_{j=1}^{k} J^{\infty}\left(w^{j}\right)
$$

and

$$
\bar{B}^{2}=\left\|(-\Delta)^{\frac{\alpha}{2}} u_{0}\right\|_{2}^{2}+\sum_{j=1}^{k}\left\|(-\Delta)^{\frac{\alpha}{2}} w^{j}\right\|_{2}^{2} .
$$

If $k=0$, we are done. If $k \geq 1$, assume by contradiction that $u_{0} \neq 0$. Then, as in Lemma 6.1,

$$
J\left(u_{0}\right)>\frac{b \bar{B}^{2}}{4} \int_{\mathbb{R}^{N}}\left|(-\Delta)^{\frac{\alpha}{2}} u_{0}\right|^{2} d x,
$$

for each $j$ there is $t_{j} \in(0,1]$ such that $I^{\infty}\left(w^{j}\left(\cdot / t_{j}\right)\right) \geq c_{1}$, which is strict if $V(x) \not \equiv V_{\infty}$, and

$$
J^{\infty}\left(w^{j}\right) \geq c_{1}+\frac{b \bar{B}^{2}}{4} \int_{\mathbb{R}^{N}}\left|(-\Delta)^{\frac{\alpha}{2}} w^{j}\right|^{2} d x
$$


where the inequality is strict if $V(x) \not \equiv V_{\infty}$. Then by formulas (6.8)-(6.9) and $\nu \leq c_{1}$, we get

$$
c_{1}+\frac{b \bar{B}^{4}}{4} \geq \nu+\frac{b \bar{B}^{4}}{4}=J\left(u_{0}\right)+\sum_{j=1}^{k} J^{\infty}\left(w^{j}\right)>k c_{1}+\frac{b \bar{B}^{4}}{4},
$$

a contradiction. Hence $u_{0}=0$ and $k=1$, in which case a contradiction follows as in the proof of Lemma 6.1. The proof is complete.

\section{REFERENCES}

[1] C. O. Alves, F. Corrêa, On existence of solutions for a class of problem involving a nonlinear operator, Appl. Nonlinear Anal. 8 (2001), 43-56. 2

[2] V. Ambrosio, T. Isernia, A multiplicity result for a fractional Kirchhoff equation in $\mathbb{R}^{N}$ with a general nonlinearity, preprint. 2, 4

[3] G. Autuori, A. Fiscella, P. Pucci, Stationary Kirchhoff problems involving a fractional elliptic operator and a critical nonlinearity, Nonlinear Anal. 125 (2015), 699-714. 2

[4] A. Azzollini, The elliptic Kirchhoff equation in $\mathbb{R}^{N}$ perturbed by a local nonlinearity, Differential Integral Equat. 25 (2012), 543-554. 2

[5] A. Baernstein, A unified approach to symmetrization. In Partial differential equations of elliptic type (Cortona, 1992), 47-91. Symposia Mathematica 35 Cambridge University Press, Cambridge, 19948

[6] H. Berestycki, P. Lions, Nonlinear scalar field equations. I. Existence of a ground state, Arch. Ration. Mech. Anal. 82 (1983), 313-345. 2

[7] H. Brezis, L. Nirenberg, Positive solutions of nonlinear elliptic problems involving critical Sobolev exponent, Comm. Pure Appl. Math. 36 (1983), 437-477. 4

[8] L. Caffarelli, L. Silvestre, An extension problem related to the fractional Laplacian, Comm. PDE 32 (2007), 1245-1260. 2

[9] M. Chipot, B. Lovat, Some remarks on nonlocal elliptic and parabolic problems, Nonlinear Anal. 30 (1997), 4619-4627. 2

[10] X. Chang, Z. Wang, Ground state of scalar field equations involving a fractional Laplacian with general nonlinearity, Nonlinearity 26 (2013), 479-494. 2, 12, 17, 21

[11] S. Dipierro, G. Palatucci, E. Valdinoci, Existence and symmetry results for a Schrödinger type problem involving the fractional laplacian, Matematiche LXVIII, (2013), 201-216. 2

[12] P. Felmer, A. Quaas, J. Tan, Positive solutions of nonlinear Schrödinger equation with the fractional Laplacian, Proc. Roy. Soc. Edinburgh Sect. A 142 (2012), 1237-1262. 2, 6

[13] A. Fiscella, E. Valdinoci, A critical Kirchhoff type problem involving a nonlocal operator, Nonlinear Anal. 94 (2014), 156-170. 2, 4

[14] R. Frank, E. Lenzmann, Uniqueness and non degeneracy of ground states for $(-\Delta)^{s} Q+Q-Q^{\alpha+1}=0$ in $\mathbb{R}$, Acta Math. 210 (2013), 261-318. 2

[15] X. He, W. Zou, Existence and concentration behavior of positive solutions for a Kirchhoff equation in $\mathbb{R}^{3}, \mathrm{~J}$. Differential Equations 252 (2012), 1813-1834. 2

[16] L. Jeanjean, On the existence of bounded Palais-Smale sequence and application to a Landesman-Lazer type problem set on $\mathbb{R}^{N}$, Proc. Roy. Soc. Edinburgh Sect. A 129 (1999), 787-809. 4, 6

[17] G. Kirchhoff, Mechanik, Teubner, Leipzig, 1883. 2

[18] N. Laskin, Fractional Schrödinger equation, Phy. Rev. E 66, 05618. 2

[19] G. Li, H. Ye, Existence of positive solutions for nonlinear Kirchhoff type problems in $\mathbb{R}^{3}$ with critical Sobolev exponent and sign-changing nonlinearities, Math. Methods Appl. Sci. 37(16) (2014), 2570-2584. 4

[20] G. Li, Y. He, Existence of positive ground state solutions for the nonlinear Kirchhoff type equations in $\mathbb{R}^{3}, \mathrm{~J}$. Differential Equations 257 (2014), 566-600. 2, 3, 4

[21] J. Lions, On some questions in boundary value problems of mathematical physics, In: Contemporary Developments in Continuum Mechanics and Partial Differential Equations. Proc. Internat. Sympos. Inst. Mat. Univ. Fed. Rio de Janeiro, (1997) In: North-Holland Math. Stud. 30 (1978), 284-346. 2

[22] Z. Liu, S. Guo, Existence and concentration of positive ground states for a Kirchhoff equation involving critical Sobolev exponent, Z. Angew. Math. Phys. 66 (2015), 747-769. 4

[23] Z. Liu, S. Guo, Existence of positive ground state solutions for Kirchhoff type problems, Nonlinear Anal. 120 (2015), 1-13. 2 
[24] T. Ma, J. Rivera, Positive solutions for a nonlinear nonlocal elliptic transmission problem, Appl. Math. Lett. 16 (2003), 243-248. 2

[25] N. Nyamoradi, Existence of three solutions for Kirchhoff nonlocal operators of elliptic type, Math. Commun. 18 (2013), 489-502. 2

[26] K. Perera, Z. Zhang, Nontrivial solutions of Kirchhoff-type problems via the Yang index, J. Differential Equations 221 (2006), 246-255. 2

[27] P. Pucci, S. Saldi, Critical stationary Kirchhoff equations in $\mathbb{R}^{N}$ involving nonlocal operators, Rev. Mat. Iberoam 32, (2016) 1-22. 2, 4

[28] P. Pucci, M. Xiang, B. Zhang, Existence and multiplicity of entire solutions for fractional p-Kirchhoff equations, Adv. Nonlinear Anal. 5 (2016), 27-55. 2

[29] S. Secchi, Ground state solutions for nonlinear fractional Schrödinger equations in $\mathbb{R}^{N}$, J. Math. Phys. 54 (2013), 031501. 2, 6

[30] R. Servadei, E. Valdinoci, The Brezis-Nirenberg result for the fractional Laplacian, Trans. Amer. Math. Soc. 367 (2015), 67-102. 2, 10

[31] L. Silvestre, Hölder estimates for solutions of integro-differential equations like the fractional Laplace, Indiana Univ. J. Math. 55 (2006), 1155-1174. 2

[32] M. Squassina, On the Struwe-Jeanjean-Toland monotonicity trick, Proc. Roy. Soc. Edinburgh Sect. A 142 (2012), 155-169. 4, 7

[33] K. Teng, Existence of ground state solutions for the nonlinear fractional Schrödinger-Poisson system with critical Sobolev exponent, J. Differential Equations, 261 (2016) 3061-3106. 3

[34] J. Van Schaftingen, Symmetrization and minimax principles, Commun. Contemp. Math. 7(2005), 463-481. 8

[35] X. Wu, Existence of nontrivial solutions and high energy solutions for Schrödinger-Kirchhoff-type equations in $\mathbb{R}^{N}$, Nonlinear Anal. RWA. 12 (2011), 1278-1287. 2

[36] M. Xiang, B. Zhang, X. Guo, Infinitely many solutions for a fractional Kirchhoff type problem via Fountain Theorem, Nonlinear Anal. 120 (2015), 299-313. 2, 4

[37] Z. Zhang, K. Perera, Sign changing solutions of Kirchhoff type problems via invariant sets of descent flow, J. Math. Anal. Appl. 317 (2006), 456-463. 2

[38] J. J. Zhang, W. M. Zou, A Berestycki-Lions theorem revisted, Commun. Contemp. Math. 14 (2012), 14 pages. 3

[39] J. J. Zhang, W. M. Zou, The critical case for a Berestycki-Lions theorem, Science China Math. 14 (2014), 541-554. 4

(Z. S. Liu)

School of Mathematics and Physics, University of South China

Hengyang, Hunan 421001, P.R. China

E-mail address: liuzhisu183@sina.com

(M. Squassina)

Dipartimento di Matematica e Fisica, Universita Cattolica del Sacro Cuore,

Via Musei 41, 25121 Brescia, ItAly

E-mail address: marco.squassina@unicatt.it

(J. J. Zhang)

College of Mathematics and Statistics, Chongqing Jiaotong University

Chongqing 400074, PR China

E-mail address: zhangjianjun09@tsinghua.org.cn 